\title{
Semiclassical collision theory. Application of multidimensional uniform approximations to the atom-rigid-rotor system*
}

\author{
H. Kreek, R. L. Ellis, and R. A. Marcus \\ Department of Chemistry, University of Illinois, Urbana, Illinois 61801 \\ (Received 26 September 1974)

\begin{abstract}
The multidimensional Bessel and Airy uniform approximations developed earlier in this series for the semiclassical $S$ matrix are applied to the atom rigid-rotor system. The need is shown for (a) using a geoemetrical criterion for determining whether a stationary phase point (s.p.pt) is a maximum, minimum, or saddle point; (b) choosing a proper quadrilateral configuration of the s.p. pts. with the phases as nearly equal as possible; and (c) choosing a unit cell to favor near-separation of variables. (a) and (b) apply both to the Airy and to the Bessel uniform approximations, and (c) to the Bessel. The use of a contour plot both to understand and to facilitate the search in new cases is noted. The case of real and complex-valued stationary phase points is also considered, and the Bessel uniform-in-pairs approximation is applied. Comparison is made with exact quantum results. As in the one-dimensional case, the Bessel is an improvment over the Airy for " $k=0$ " transitions, while for other transitions they give similar results. Comparison in accuracy with the results of the integral method is also given. As a whole, the agreement can be considered to be reasonable. The improvement of the present over various more approximate results is shown.
\end{abstract}

\section{INTRODUCTION}

In the preceding paper of this series a multidimensional Bessel uniform approximation was developed for the semiclassical $S$ matrix. ${ }^{1}$ In the present paper this approximation is applied and compared with the previously developed Airy approximation ${ }^{2}$ and with exact results. ${ }^{3}$ For concreteness the applications are made to the atom-homonuclear rigid rotor problem. The typical configurations, and their origin, of the stationary phase points in the $S$ matrix integral expression are discussed in Sec. II, the procedure for applying the uniform approximations in Sec. III, and the numerical results and comparison with exact results in Sec. IV.

The Hamiltonian used in the present work is a standard one (e.g., Refs. 4, 5). It is given in Appendix A for completeness, together with the coordinates and a definition of the other symbols and parameters. For Secs. II-IV it suffices to note that $\hat{l}, \hat{j}$, and $\hat{j}$ are the orbital, rotational, and total angular momenta; $l, j$, and $J$ are the corresponding quantum numbers. Classically, $l$ and $j$ vary continuously along a trajectory in the interaction region. Their final values for arbitrary trajectories are $l^{f}$ and $j^{f}$. J is an integer and remains constant. At the stationary phase points of the phase $\Delta$ in an integral expression for the $S$ matrix for a transition $\left(l_{1}, j_{1}\right) \rightarrow\left(l_{2}, j_{2}\right), l^{f}$ and $j^{f}$ take on the desired final integer values $l_{2}$ and $j_{2}$.

Contour plots in the present paper are expressed in terms of certain derived coordinates ${ }^{6,7} \bar{q}_{j}^{0}$ and $\bar{q}_{i}^{0}$, canonically conjugate to $\hat{j}_{1}$ and $\hat{l}_{1},{ }^{6}$ the initial values of $\hat{j}$ and $\hat{l}$, respectively, and defined in Appendix $A$. The original coordinates, also conjugate to $\hat{j}_{1}$ and $\hat{l}_{1}$, are the angle variables $q_{j}$ and $q_{l}$. The radial coordinate for the colliding pairs is $R$ and its conjugate momentum is $P_{R}$. Derived coordinates canonically conjugate ${ }^{6}$ to the final values of $\hat{l}$ and $\hat{j}$ are denoted by $\bar{q}_{l}^{f}$ and $\bar{q}_{j}^{f}$. While $q_{l}$, $q_{j}$, and $R$ vary during a collision, $\bar{q}_{l}^{0}, \bar{q}_{j}^{0}, \bar{q}_{l}^{f}$, and $\bar{q}_{j}^{f}$ are constants of the motion.

\section{NUMBER AND CONFIGURATION OF STATIONARY PHASE POINTS}

In order to apply the multidimensional uniform Bessel and Airy approximations developed in the previous papers of this series ${ }^{1,2}$ to the semiclassical expression for the $S$ matrix it is necessary to determine the stationary phase points. These are the values of $\left(q_{l}^{0}, \bar{q}_{j}^{0}\right)$ characterizing trajectories which, for the present problem, yield values of $l^{f}$ and $j^{f}$ satisfying simultaneously

$$
l^{f}-l_{2}=0 \text { and } j^{f}-j_{2}=0 \text {. }
$$

If contours of constant $l^{f}$ are drawn in a $\left(\bar{q}_{l}^{0}, \bar{q}_{j}^{0}\right)$ space, and if those of constant $j^{f}$ are also drawn, the stationary phase points are those for which the contour $l^{f}=l_{2}$ intersects the one for $j^{f}=j_{2}$. To obtain some insight into the number of stationary points and their configuration, it is useful to consider first these $l^{f}$ and $j^{f}$ contours for several varied cases. The contour plots (Figs. $1-3$, given later) were obtained from a smooth interpolation of a $10 \times 10$ grid of points in the $\left(q_{l}^{0} . \bar{q}_{j}^{0}\right)$ space. ${ }^{8}$

In actual problems the configuration of the stationary phase points in the $\left(\bar{q}_{l}^{0}, \bar{q}_{j}^{0}\right)$ space is dependent on various factors, including the initial orientation of the orbital and rotational angular momentum vectors $\hat{\mathfrak{l}}_{1}$ and $\hat{\mathrm{j}}_{1}$, respectively. For illustration it is useful to consider first the extreme but simple case of motion in a plane. Here, the initial $\hat{\mathrm{l}}$ and $\hat{\mathrm{j}}$ may be parallel or antiparallel, and so the factor $\left(\hat{l}^{2}+\hat{j}^{2}-\hat{J}^{2} / 2 \hat{l} \hat{j}\right)$ in Eq. (A4) (Appendix A) which is the cosine of the angle between the $\hat{I}$ and $\hat{j}$ planes, equals +1 or -1 , respectively. One can then show that the only angle variable which influences the inelasticity is $\bar{q}_{l}^{0}-\bar{q}_{j}^{0}$, when $\hat{\mathrm{l}}$ and $\hat{\mathrm{j}}$ are parallel, or $\bar{q}_{l}^{0}$ $+\bar{q}_{j}^{0}$ when $\hat{1}$ and $\hat{j}$ are antiparallel. In this $\left(\bar{q}_{l}^{0}, \bar{q}_{j}^{0}\right)$ space one may draw curves of constant $\hat{j}^{f}$. (Since $\hat{l}^{f}$ equals $\hat{J}^{f}-\hat{j}^{f}$ in this planar case, these curves are also curves of constant $\hat{l}^{f}$.)

In the present case the curves of constant $\hat{j}^{f}$ are seen 
from the above discussion to be straight lines, inclined at a slope of $45^{\circ}$ to the $\bar{q}_{l}^{0}$ axis, and having a positive slope when $\hat{j}_{1}$ and $\hat{l}_{1}$ are parallel and a negative one when they are antiparallel. Because $\hat{j}^{f}$ thus depends only on one coordinate, namely on the coordinate normal to the family of parallel straight lines, a stationary phase point (or really line here) occurs at the value of this coordinate where $\hat{j}^{f}$ equals the desired final value $\hat{j}_{2}$ for a transition $\left(l_{1}, j_{1}\right)-\left(l_{2}, j_{2}\right)$. In this planar case, $l_{2}$ is then automatically determined from the algebraic equation $\hat{j}_{2}+\hat{l}_{2}=\hat{J}_{1}=\hat{J}_{2}=\hat{J}^{f}$. The lines of the final $j^{f}$ and $l^{f}$ intersect along a "line" (i. e., coincide), rather than at a point.

If now $\hat{\mathrm{j}}_{1}$ and $\hat{\mathrm{l}}_{1}$ are neither parallel nor antiparallel, but almost so, the lines of constant $j^{f}$ (and those of constant $l^{f}$ ) are no longer straight, but almost so. For example, in Fig. 1 the contours of (a) constant $l^{f}$ and (b) constant $j^{f}$ are given. In these two figures $\left(J_{1}, l_{1}, j_{1}\right)$ is $(16,6,10)$. Since $\left|\hat{j}_{1}\right|,\left|\hat{l}_{1}\right|$, and $\left|\hat{j}_{1}\right|$ are respectively equal to $16 \frac{1}{2}, 6 \frac{1}{2}$, and $10 \frac{1}{2}$ semiclassically, ${ }^{9}$ this case is almost (but not quite) a planar one $\left(16 \frac{1}{2} \neq 6 \frac{1}{2}+10 \frac{1}{2}\right)$. The contours of constant $l^{f}$ can be termed "lines of translation" in this example [Fig. 1(a)], since they do not close on themselves and form "ellipses." In Fig. 1(b) the contour for $j^{f}=12.0$ forms an ellipselike curve, and can be termed a "line of libration," while the contours for $j^{f}=8.0$ and 10.0 are lines of translation. A separatrix would separate each family of lines of translation from each family of concentric “ellipses".

With increasing departure from coplanarity of $\hat{\mathbb{1}}_{1}$ and $\hat{\mathrm{j}}_{1}$ [as in Figs. 2(a) and 2(b) where $\left(J_{1}, l_{1}, j_{1}\right)$ is $(18,16$, 4)], the lines of translation, which tended to parallel the straight lines of the coplanar case, have given way increasingly to closed curves. When the departure from coplanarity is still larger [e.g., when $\hat{j}_{1}$ and $\hat{i}_{1}$ are approximately perpendicular to each other, as is the case in Figs. 3(a) and $3(\mathrm{~b})$ where $\left(J_{1}, l_{1}, j_{1}\right)$ is $\left.(6,4,4)\right]$, the major axes of the "ellipses" and the lines of translation are no longer inclined nearly $45^{\circ}$ to the $\bar{q}_{l}^{0}$ axis, with either positive or negative slopes. Their slopes are rather somewhere in between.

As al ready noted, the points of stationary phase for the transition $\left(l_{1}, j_{1}\right) \rightarrow\left(l_{2}, j_{2}\right)$ occur when a contour of $j^{f}=j_{2}$ intersects a contour of $l^{f}=l_{2}$. When both sets of contours are lines of translation (rather than of libration), there are typically four stationary phase points: Because of a periodic roughly sinusoidal dependence of $j^{f}$ on the coordinate normal to the lines of translation, the contour lines for which $j^{f}$ equals $j_{2}$ occur in pairs as in Fig. 1(b). Similarly, contour lines for which $l^{f}$ equals $l_{2}$ occur in pairs as in Fig. 1(a). The first pair intersects the second at four real points, if they intersect at all, in the cases examined.

On the other hand, if the contour $j^{f}=j_{2}$ is an ellipselike closed curve, and if the one $l^{f}=l_{2}$ is also an "ellipse," then these two "ellipses" intersect, in the cases examined, at only two real stationary phase points. There are also complex stationary phase points, of course, and we have located them in most of the cases investigated here. Their effect is given later in Table II.

Sometimes, particularly in a transition in which $j_{2}$ and $l_{2}$ equal $j_{1}$ and $l_{1}$, respectively, a contour of constant $j^{f}=j_{2}$ tends to hover around the contour for constant $l^{f}=l_{2}$, making the stationary phase points difficult to find. This behavior, which we have previously called a "clinging vine" case, ${ }^{10}$ gives rise to a high transition probability (e.g. , Table I of Ref. 10). It would be disastrous for any Airy uniform approximation, but might be accessible to a modified Bessel uniform. We postpone treating this case by the uniform method. We have, however, treated it by the integral method in an earlier paper. ${ }^{10}$
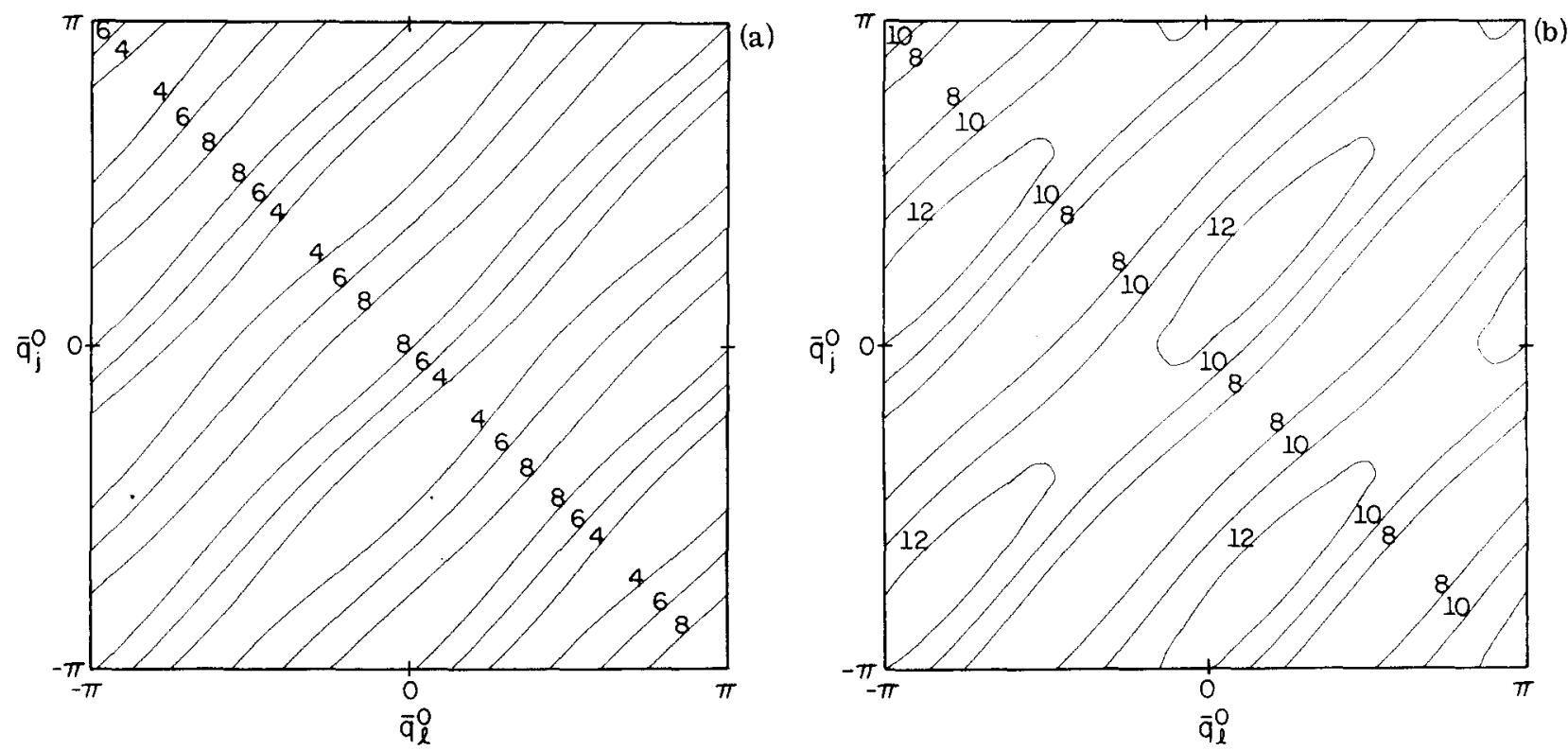

FIG. 1. (a) Contours of constant $l^{f}$ in the space $\left(\bar{q}_{l}^{0}, \bar{q}_{j}^{0}\right)$ for the $S$ matrix $S_{l_{2}, j_{2} ; 6,10}^{16}$. Only integer values of $l^{f}$ are indicated, but contours for all possible $l^{f}$ 's can be drawn. Analogous remarks apply to Figs. $1(\mathrm{~b})-3(\mathrm{~b})$. (b) Contours of constant $j^{f}$ in the space $\left(\bar{q}_{l}^{0}, \bar{q}_{j}^{0}\right)$ for the $S$ matrix $S_{l_{2}, j 2 ; 6,10^{\circ}}^{16}$ 

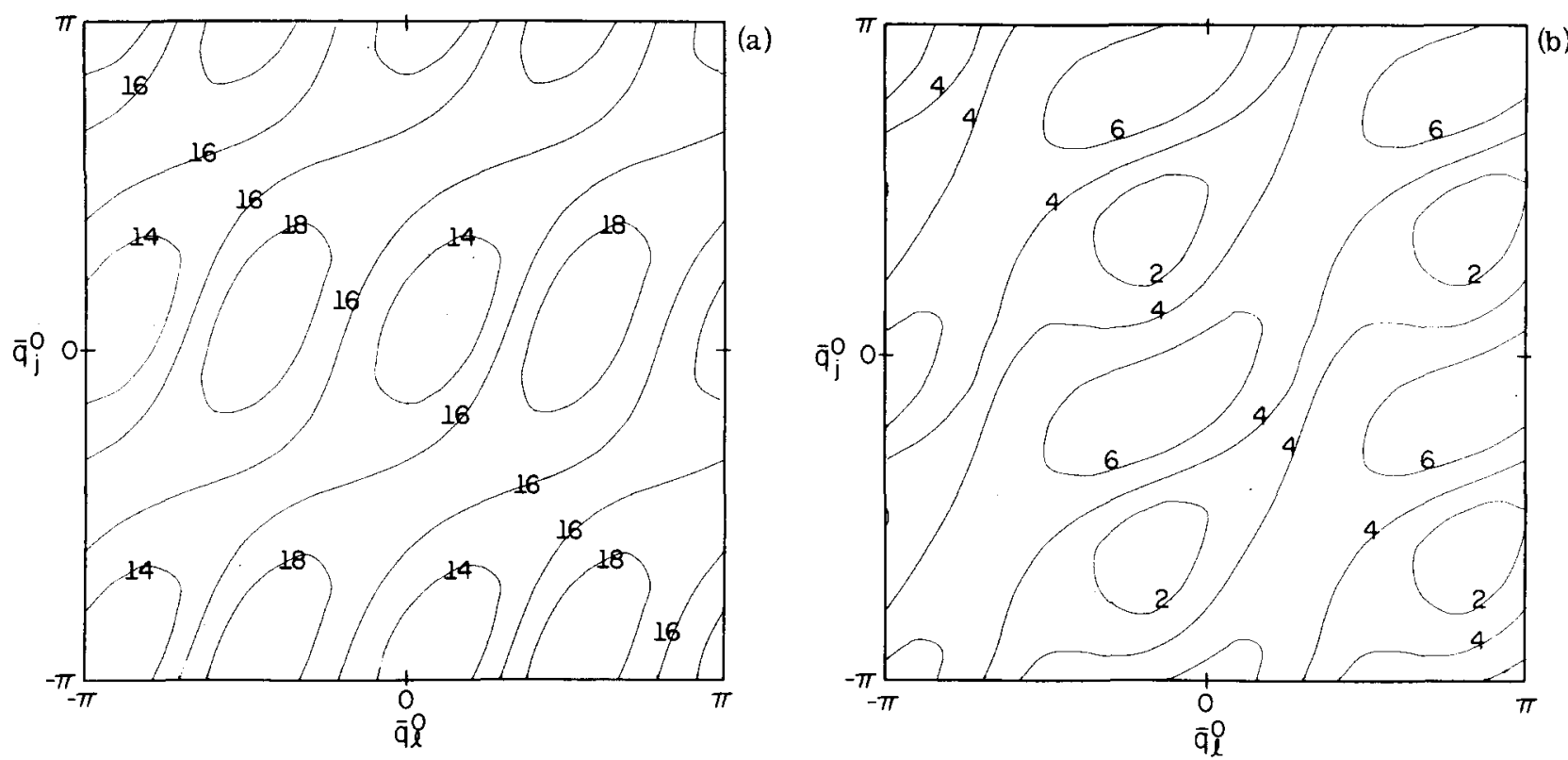

FIG. 2. (a) Contours of constant $l^{f}$ in the space $\left(\bar{q}_{l}^{0}, \bar{q}_{j}^{0}\right)$ for the $S$ matrix $S_{l_{2}, j_{2} ; 16,4}^{18}$; (b) contours of constant $j^{f}$ in the space $\left(\bar{q}_{l}^{0}, \bar{q}_{j}^{0}\right)$ for the $S$ matrix $S_{l_{2}, j_{2} ; 16,4}^{18}$

\section{PROCEDURE FOR APPLYING PSC, BESSEL, AND AIRY FORMALISMS}

In terms of the notation given by Eq. (3.5) of Ref. 10, the $S$-matrix element for a transition $\left(l_{1}, j_{1}\right) \rightarrow\left(l_{2}, j_{2}\right)$ is

$$
S_{l_{2}, j_{2} ; l_{1}, j_{1}}^{J}=(2 \pi)^{-2} \int_{-\pi}^{\pi} \int_{-\pi}^{\pi} G^{1 / 2} e^{i \Delta} d \bar{q}_{l}^{0} d \bar{q}_{j}^{0},
$$

where $G$ is the Jacobian for the dynamical transformation $\left(\bar{q}_{l}^{0}, \bar{q}_{j}^{0}\right)-\left(\bar{q}_{l}^{f}, \bar{q}_{j}^{f}\right)$. It is a signed determinant, i. e. , has a phase.

$$
G=\left|\partial\left(\bar{q}_{l}^{f}, \bar{q}_{j}^{f}\right) / \partial\left(\bar{q}_{l}^{0}, \bar{q}_{j}^{0}\right)\right| .
$$

$\Delta$ is given by (3.3) below, and the other symbols were defined in Sec. I.

$$
\begin{aligned}
\Delta= & \left(l^{f}-l_{2}\right) \bar{q}_{l}^{f}-\int_{l_{1}}^{l^{f}} q_{l}(t) d l(t)+\left(j^{f}-j_{2}\right) \bar{q}_{j}^{f}-\int_{j_{1}}^{j^{f}} q_{j}(t) d j(t) \\
& -\frac{1}{\hbar_{0}} \int_{P_{R_{1}}}^{P_{R}^{f}} R(t) d P_{R}(t)+\frac{1}{2} \pi\left(l_{1}+l_{2}+1\right) .
\end{aligned}
$$

( $P_{R_{1}}$ is the initial value of $\left.P_{R^{*}}\right)$ The $q_{t}(t), q_{j}(t), l(t)$, $j(t)$, and $R(t)$ in the integrands in (3.3) denote instantaneous values of the relevant coordinates and momenta. $\hbar_{0}$ is a dimensionless quantity defined in Appendix A.

For the following analysis we write the phase $\Delta$ in (3.3) as the sum of two terms, $\Delta_{L}$ and $\Delta_{P}$ :
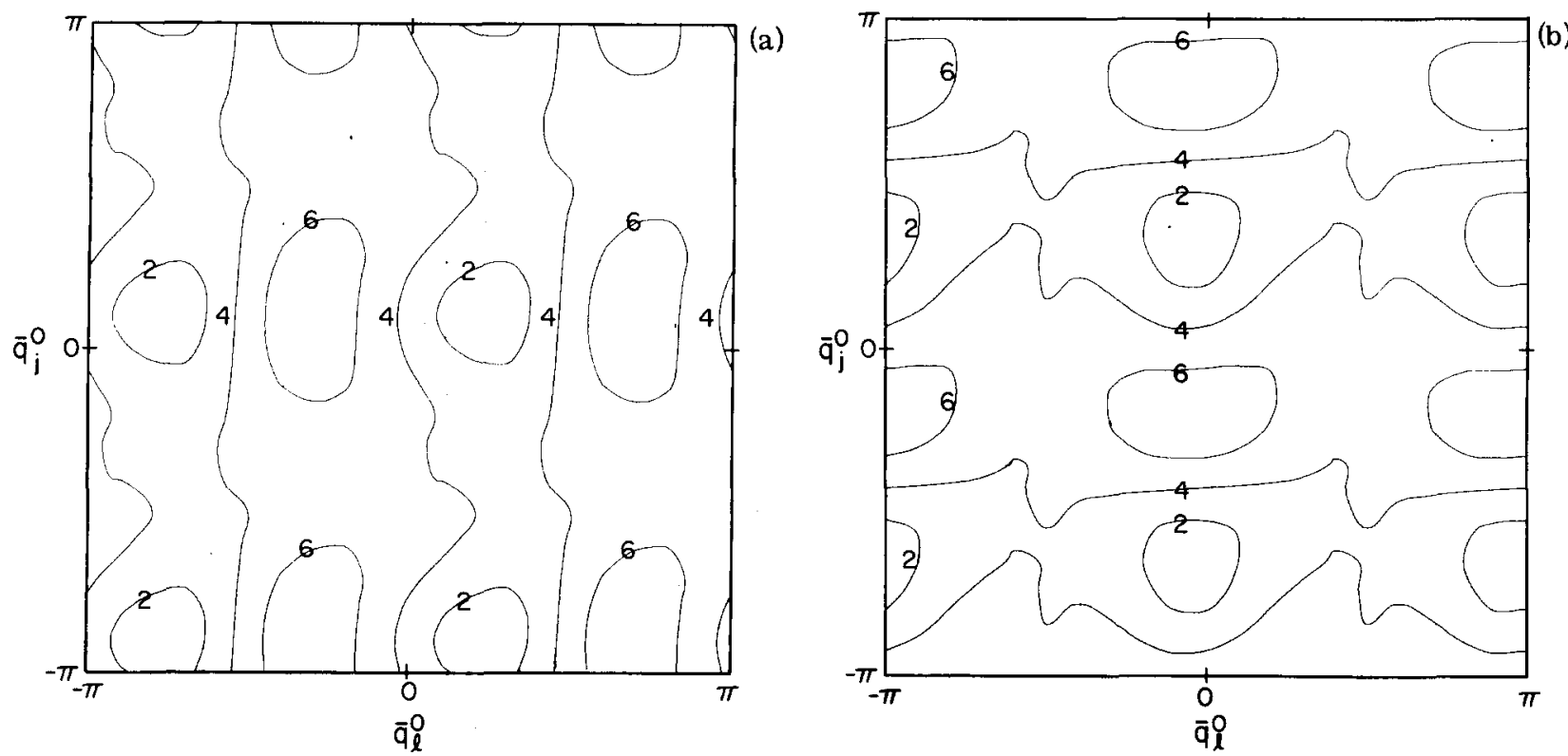

FIG. 3. (a) Contours of constant $l^{f}$ in the space $\left(\bar{q}_{l}^{0}, \bar{q}_{j}^{0}\right)$ for the $S$ matrix $S_{l_{2} j_{2} ; 4,4}^{6}$ (b) contours of constant $j^{f}$ in the space $\left(\bar{q}_{l}^{0}\right.$, $\left.\bar{q}_{j}^{0}\right)$ for the $S$ matrix $S_{l_{2}, j_{2} ; 4,4}^{6}$. 


$$
\begin{aligned}
\Delta= & \Delta_{L}+\Delta_{P} \\
\Delta_{L}= & \left(l_{1}-l_{2}\right) \bar{q}_{l}^{f}+\left(j_{1}-j_{2}\right) \bar{q}_{j}^{f} \\
\Delta_{P}= & \left(l^{f}-l_{1}\right) \bar{q}_{l}^{f}-\int_{l_{1}}^{l^{f}} q_{l}(l) d l(t)+\left(j^{f}-j_{1}\right) \bar{q}_{j}^{f}-\int_{j_{1}}^{j^{f}} q_{j}(t) d j(t) \\
& -\frac{1}{\hbar_{0}} \int_{P_{R_{1}}}^{P_{R}^{f}} R(t) d P_{R}(l)+\frac{1}{2} \pi\left(l_{1}+l_{2}+1\right)
\end{aligned}
$$

$\Delta_{P}$, and hence $\exp i \Delta_{p}$, can be shown to be a periodic function of $\bar{q}_{l}^{0}$ and $\bar{q}_{j}^{0}$ with a period equal to that of the unit cell (i.e., $2 \pi$; or in the case of a collision of an atom and a homonuclear molecule, $\pi$ ). ${ }^{11 a} \Delta_{L}$ is a linear function of $\bar{q}_{l}^{f}$ and of $\bar{q}_{j}^{f}$, but expis$\Delta_{L}$ is a periodic function of $\bar{q}_{i}^{0}$ and $\bar{q}_{j}^{0} .{ }^{11 \mathrm{~b}}$ Thus, expis is a periodic function of $\bar{q}_{l}^{0}$ and $\bar{q}_{j}^{0}$.

While $\Delta_{L}$ is a linear function of $\bar{q}_{l}$ and $\bar{q}_{j}^{f}$, it is not a linear function of $\bar{q}_{l}^{0}$ and $\bar{q}_{j}^{0}$. It can be written as the sum of a linear function of $\bar{q}_{l}^{0}$ and $\bar{q}_{j}^{0}, \Delta_{L}^{\prime}$, and of a periodic function of those variables ${ }^{12}$ :

$$
\Delta_{L}^{\prime}=\left(l_{1}-l_{2}\right) \bar{q}_{i}^{0}+\left(j_{1}-j_{2}\right) \bar{q}_{j}^{0} .
$$

$\Delta$ itself is seen to be the sum of a linear and a periodic function of $\bar{q}_{l}^{0}$ and $\bar{q}_{j}^{0}$.

The location of the stationary phase points in $\mathrm{Eq} .(3.1)$ was obtained by a curve crawling procedure that will be described elsewhere. ${ }^{13}$ Because of the symmetry of the homonuclear diatomic molecule, the area of search for points of stationary phase can be restricted ${ }^{14,4,10}$ to a $\pi$-interval in the variable $\bar{q}_{l}^{0}$ and to a $\pi$-interval in $\bar{q}_{j}^{0}$. For the classical and primitive semiclassical probabilities, the phases obtained from the trajectories can be used as calculated [Eqs. (B1)-(B3) of Appendix B].

\section{A. Case of four real stationary phase points}

For both the Airy and Bessel formulations one must choose a $\pi^{2}$ region so that when there are four points of stationary phase they conform to the configuration on which concepts and resulting formulas are based. The

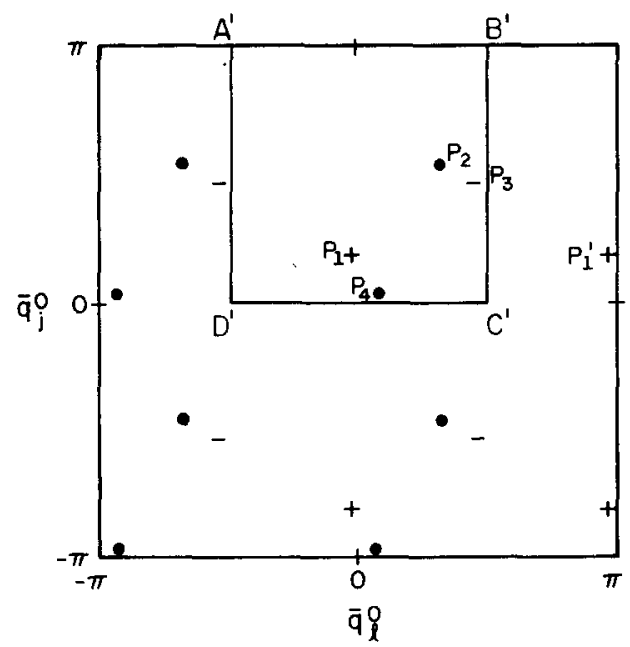

FIG. 4. Arrangement of stationary phase points in the space $\left(\bar{q}_{3}^{0}, \bar{q}_{j}^{0}\right)$ for the $S$ matrix $S_{4,12 ; 6,10 .}^{16}$ Maximum $(+)$, minimum $(-)$, saddle points $(\bullet)$. The Airy uniform approximation for the $S$ matrix can be used over the unit cell $A^{\prime} B^{\prime} C^{\prime} D^{\prime}$.

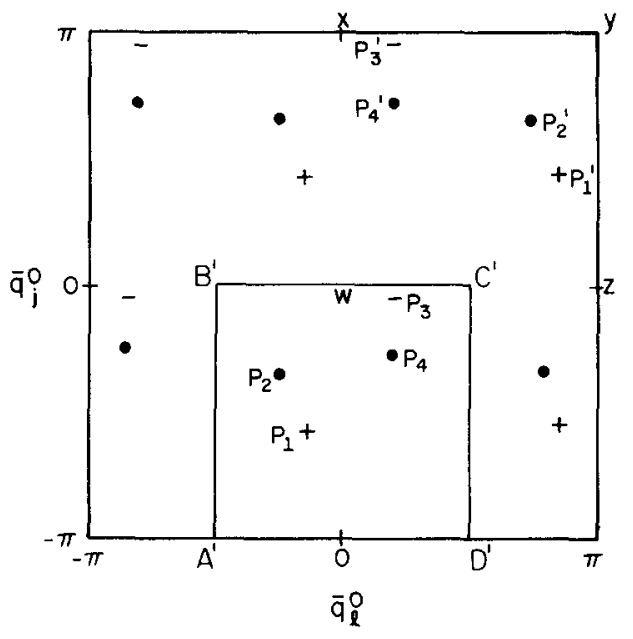

FIG. 5. Arrangement of stationary phase points in the space $\left(\bar{q}_{l}^{0}, \bar{q}_{j}^{0}\right)$ for the $S$ matrix $S_{8,8 ; 6,10}^{16}$. Maximum $(+)$, minimum $(-)$, saddle points $(\bullet)$. The Airy uniform approximation for the $S$ matrix can be used over the unit cell $A^{\prime} B^{\prime} C^{\prime} D^{\prime}$.

four points should be at the corners of a quadrilateral, with the maximum and the minimum at opposite corners, and with the two saddle points at the other pair of opposite corners. The procedure used in performing calculations is outlined below.

After the position of the stationary points and the value of $\Delta$ and $G$ at each of these points has been determined by the curve crawling procedure, the maximum, minimum, and saddle points can each be identified unequivocably using the following properties of the Hessian of a function $\Delta(x, y)\left[\text { i. e., of } \Delta\left(\bar{q}_{l}^{0}, \bar{q}_{j}^{0}\right) \text { in }(3.3)\right]^{15}$ :

$\frac{\partial^{2} \Delta}{\partial x^{2}} \frac{\partial^{2} \Delta}{\partial y^{2}}-\frac{\partial^{2} \Delta}{\partial x \partial y}>0, \frac{\partial^{2} \Delta}{\partial x^{2}}<0, \frac{\partial^{2} \Delta}{\partial y^{2}}<0$ (at a maximum); $\frac{\partial^{2} \Delta}{\partial x^{2}} \frac{\partial^{2} \Delta}{\partial y^{2}}-\frac{\partial^{2} \Delta}{\partial x \partial y}>0, \frac{\partial^{2} \Delta}{\partial x^{2}}>0, \frac{\partial^{2} \Delta}{\partial y^{2}}>0$ (at a minimum);

$\frac{\partial^{2} \Delta}{\partial x^{2}} \frac{\partial^{2} \Delta}{\partial y^{2}}-\frac{\partial^{2} \Delta}{\partial x \partial y}<0$ (at a saddle point).

(The maximum, minimum, etc. cannot be identified by their relative values of $\Delta$, since the latter includes a linear function of $\bar{q}_{l}^{0}$ and $\bar{q}_{j}^{0}$ and so has an infinite number of values.)

An example (and there are many) where the four stationary phase points in the interval $\left(0 \leq \bar{q}_{l}^{0} \leq \pi\right)$ and $(0$ $\left.\leq \vec{q}_{j}^{0} \leq \pi\right)$ did not occur in the correct configuration is given in Fig. 4. The data in this figure were obtained in the calculation of $S_{4,12 ; 6,10}^{16}$ (denoting $S_{l_{2}, j_{2} ; l_{1}, j_{1}}^{J}$ ) and the cited area interval is the upper right quadrant. The maximum $P_{1}^{\prime}$ and minimum $P_{3}$ are not at opposite corners of a quadrilateral. Moreover, the relative $\Delta$ values of the four points were not consistent with the identification of the maximum, etc., based on Eq. (3.8).

Because of the periodic array of stationary-phase points in $\left(q_{l}^{0}, \bar{q}_{j}^{0}\right)$ space it sufficed to select in Fig. 4 another $\pi^{2}$ area, merely by shifting the horizontal and vertical boundaries, so as to obtain four points in the correct configuration. A new $\pi^{2}$ area $A^{\prime} B^{\prime} C^{\prime} D^{\prime}$ is defined by this new unit cell, and the relative $\Delta$ values now agree with the identification of maxima, minima, 
and saddle points provided by the Hessian. The integral in Eq. (3.1), evaluated over the new cell, numerically equals the integral over the old cell, since to each point in the former there corresponds one point in the latter with the same value of the integrand. However, results based on the uniform approximation formula would not be equal, because of the model used for those formulas. For the example in Fig. 4, one may now use the Airy uniform approximation with the stationary phase points labeled $P_{1}, P_{2}, P_{3}$, and $P_{4}$ without any further analysis. That formula is given by Eq. (B4) of Appendix B.

Sometimes, as in Fig. 5 , the points in the $(0, \pi)^{2}$ appear to be in the correct configuration but in the $(-\pi, \pi)^{2}$ region there is another set of points, enclosed by the square $A^{\prime} B^{\prime} C^{\prime} D^{\prime}$, which is also in the correct configuration. In this case, one should select the configuration for which the four $\Delta$ 's are closest together in value. The reason is based on the topology of the $\Delta$ surface and is given in Appendix $\mathrm{C}$ : The $P_{1} P_{2} P_{3} P_{4}$ in a true unit cell can be partially bordered by regions in which $\Delta$ changes rapidly.

In the particular example of Fig. 5, which is an example of the points found for the calculation of the $S$ matrix $S_{8,8 ; 6,10}^{16}$, we have for $\Delta\left(P_{1}^{\prime}\right), \Delta\left(P_{2}^{\prime}\right), \Delta\left(P_{3}^{\prime}\right)$, and $\Delta\left(P_{4}^{\prime}\right)$ the values $-42.1769,-36.4490,-36.8283$, and -42.8364, respectively. Using Eqs. (3.4)-(3.7) the phases at certain other points $P_{1}, P_{2}, P_{3}$, and $P_{4}$ in Fig. 5 are found to be $-42.1769,-42.7322,-43.1115$, and -42.8364 , respectively. The phases at these stationary phase points are closer in value than those at the original points, and they too are in a proper quadrilateral configuration. One, therefore, chooses the new unit cell as $A^{\prime} B^{\prime} C^{\prime} D^{\prime}$ in Fig. 5, and uses the value of the phase and $G_{i}$ at $P_{1}, \ldots, P_{4}$ in the Airy approximation.

The above two main points of the analysis have not been brought out before in the literature.

To apply the Bessel formalism it is necessary to consider one further topological property of the phase $\Delta$, so as to determine the integers $k$ and $K$ appearing in the

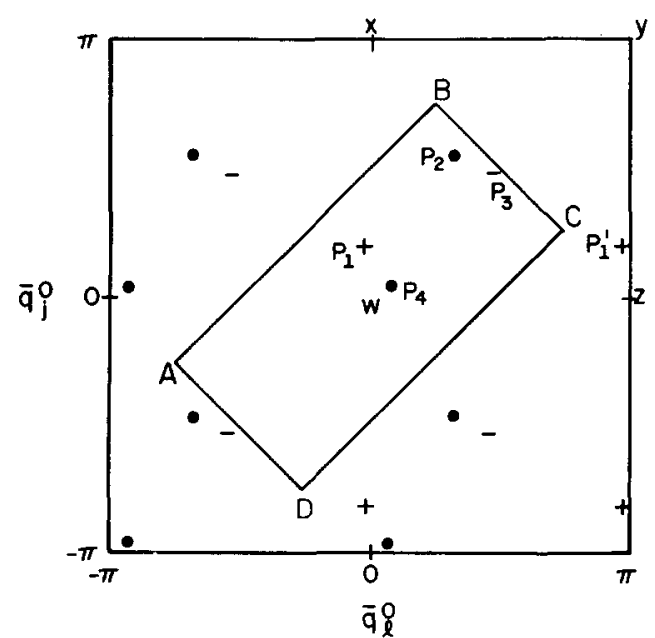

FIG. 6. Arrangement of stationary phase points in the space $\left(\bar{q}_{l}^{0}, \vec{q}_{j}^{0}\right)$ for the $S$ matrix $S_{4,12 ; 6,10^{\circ}}^{16}$ Maximum $(+)$, minimum $(-)$, saddle points $(\bullet)$. The Bessel uniform approximation for the $S$ matrix can be used over the unit cell $A B C D$.
Bessel expression, Eq. (B11) of Appendix B. Here, we must choose a newly shaped unit cell of $\pi^{2}$ area, containing the four stationary phase points not only in the proper quadrilateral configuration, but also so as to make possible a near separation of variables. To determine $k$ and $K$ one may then make use of Eqs, (3.4)-(3.7) with the definition of $k$ and $K$ as the coefficients most closely associated with points $P_{1} P_{2}$ and $P_{1} P_{4}$, respectively, in the linear term in the phase $\Delta$. Specifically, Eq. (3.9) below, derived in Appendix C [Eq. (C5)], is used. As before, calculation of $S_{4,12 ; 6,10}^{16}$, whose stationary phase points were described in Fig. 4, will be used as an example.

The original unit cell was the interval $0 \leq \bar{q}_{t}^{0} \leq \pi, 0$ $\leq \bar{q}_{j}^{0} \leq \pi$ and is labeled $W X Y Z$ in Fig. 6. As can be seen in Fig. 6, the edges $P_{1} P_{4}$ and $P_{2} P_{3}$ of the quadrilateral $P_{1} P_{2} P_{3} P_{4}$ are nearly parallel to the diagonal $X Z$ of the original unit cell. The edges $P_{1} P_{2}$ and $P_{4} P_{3}$ are nearly parallel to the diagonal $W Y$. To favor the near separation of variables a newly shaped rectangular unit cell $(A B C D$ in Fig. 6) is chosen, so as to parallel the diagonals $X Z$ and $W Y$. The longer side of the new rectangle $A B C D$ is taken $A B$, since the distance $P_{1} P_{2}$ and $P_{4} P_{3}$ exceeds $P_{2} P_{3}$ and $P_{1} P_{4}$. We choose the length of edge $A B$ equal to the length of the diagonal $W Y$. To preserve the $\pi^{2}$ area of the unit cell the length of $A D$ is therefore chosen to be one half the length of the diagonal $X Z$. (Compare also $A B C D$ in Fig. 6 with $T_{2} T_{11} T_{12} T_{7}$ in Fig. 8 in Appendix C.)

We define point $A$ to be the one which "corresponds" to the maximum, point $P_{1}$, in the "congruent" quadrilateral $P_{1} P_{2} P_{3} P_{4}$. A clockwise order for both sets of points $\left(P_{1} P_{2} P_{3} P_{4}\right.$ and $\left.A B C D\right)$ has been adopted throughout this paper, with $A$ "corresponding" to $P_{1}, B$ to $P_{2}$, etc.

From Eq. (C5) and the several lines following in Appendix $C$, we have

$$
\pi k=\left(\Delta_{L}^{\prime}\right)_{B}-\left(\Delta_{L}^{\prime}\right)_{A}, \quad \pi K=\left(\Delta_{L}^{\prime}\right)_{D}-\left(\Delta_{L}^{\prime}\right)_{A},
$$

where $\left(\Delta_{L}^{\prime}\right)_{A},\left(\Delta_{L}^{\prime}\right)_{B} \ldots$ are the values of $\Delta_{L}^{\prime}$ at the points $A, B, \ldots$. Substituting the definition of $\Delta_{L}^{\prime}$ from Eq. (3.7) into Eq. (3.9) and using the fact that in this construction $A B$ is parallel to $W Y$ and equal to it in length we obtain

$$
\begin{aligned}
\pi k= & \left(l_{1}-l_{2}\right)\left[\left(\bar{q}_{l}^{0}\right)_{A}+\pi\right]+\left(j_{1}-j_{2}\right)\left[\left(\bar{q}_{j}^{0}\right)_{A}+\pi\right] \\
& -\left(l_{1}-l_{2}\right)\left(\bar{q}_{l}^{0}\right)_{A}-\left(j_{1}-j_{2}\right)\left(\bar{q}_{j}^{0}\right)_{A},
\end{aligned}
$$

since one can see from Fig. 6 that $\left(\bar{q}_{l}^{0}\right)_{B}$ equals $\left(\bar{q}_{l}^{0}\right)_{A}+\pi$ and that $\left(\bar{q}_{j}^{0}\right)_{B}$ equals $\left(\bar{q}_{j}^{0}\right)_{A}+\pi$.

To obtain $K$ we use the fact that $A D$ is parallel to $X Z$ and is $\frac{1}{2}$ the length of $X Z$. Using Eqs. (3.7) and (3.9) we have

$$
\begin{aligned}
\pi K= & \left(l_{1}-l_{2}\right)\left[\left(\bar{q}_{l}^{0}\right)_{A}+\frac{1}{2} \pi\right]+\left(j_{1}-j_{2}\right)\left[\left(\bar{q}_{j}^{0}\right)_{A}-\frac{1}{2} \pi\right] \\
& -\left(l_{1}-l_{2}\right)\left(\bar{q}_{l}^{0}\right)_{A}-\left(j_{1}-j_{2}\right)\left(\bar{q}_{j}^{0}\right)_{A},
\end{aligned}
$$

since one can see from Fig. 6 that $\left(\bar{q}_{l}^{0}\right)_{D}$ equals $\left(\bar{q}_{l}^{0}\right)_{A}$ $+\frac{1}{2} \pi$ and that $\left(q_{j}^{0}\right)_{D}$ equals $\left(q_{j}^{0}\right)_{A}-\frac{1}{2} \pi$. Thus, in this example of $S_{4,12 ; 6,10}^{16}$ where $l_{1}-l_{2}=2$ and $j_{1}-j_{2}=-2$ we have $k=0, K=2$.

In all cases, the points $A B C D$ of the newly shaped unit 
TABLE I. Comparison of quantum with Bessel and Airy uniform approximations for rotational-translational transition probability: Four real stationary phase points in $\pi^{2}$ area.

\begin{tabular}{|c|c|c|c|c|c|}
\hline \multirow[b]{2}{*}{$J$} & \multirow[b]{2}{*}{$l_{2}, j_{2} ; l_{1}, j_{1}$} & \multicolumn{3}{|c|}{ Probabilities $^{a}$} & \multirow{2}{*}{$\begin{array}{l}\text { Bessel } \\
\text { parameter } \\
K, k\end{array}$} \\
\hline & & Quantumb & Bessel & Airy & \\
\hline \multirow[t]{4}{*}{$\overline{16}$} & $12, \quad 4 ; 10,6$ & 0.297 & 0.303 & 0.461 & 2,0 \\
\hline & 8,$8 ; 10,6$ & 0.296 & 0.300 & 0.462 & 2,0 \\
\hline & 4,$12 ; 6,10$ & 0.289 & 0.263 & 0.378 & 2,0 \\
\hline & 8,$8 ; 6,10$ & 0.285 & 0.289 & 0.442 & 0,2 \\
\hline \multirow[t]{2}{*}{6} & 2,$4 ; 4,2$ & 0.220 & 0.233 & 0.285 & 2,0 \\
\hline & 10,$4 ; 8$ & 0.297 & 0.300 & 0.482 & 0,2 \\
\hline \multirow[t]{2}{*}{14} & 12,$2 ; 10$ & 0.275 & 0.284 & 0.382 & 2,0 \\
\hline & 8,$6 ; 10$ & 0.289 & 0.295 & 0.429 & 2,0 \\
\hline
\end{tabular}

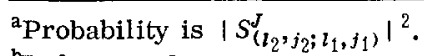

${ }^{\mathrm{b}}$ Reference 3 .

cell (e.g., Figs. 6 and 7) are chosen so that its sides lie parallel to lines passing through the periodic array of points $\left(q_{l}^{0}, \bar{q}_{j}^{0}\right)=(\cdots,-2 \pi,-\pi, 0, \pi, 2 \pi, \ldots),(. \circ,-2 \pi$, $-\pi, 0, \pi, 2 \pi, \ldots)$ and such that when $K$ and $k$ are calculated with Eq. (3.9) they are integers.

The Bessel expression, Eq. (B11) of Appendix B, for the $S$ matrix with $K=2, k=0$ and with the phases and $G_{i}$ from points $P_{1}, P_{2}, P_{3}$, and $P_{4}$ may now be used.

To perform the Bessel calculation for the example given in Fig. 5 we note that in that figure the edges $P_{1} P_{2}$ and $P_{4} P_{3}$ of the quadrilateral $P_{1} P_{2} P_{3} P_{4}$ are nearly parallel to the edge $W X$ of the original unit cell, and that the edges $P_{1} P_{4}$ and $P_{2} P_{3}$ are nearly parallel to the diagonal $W Y$. A newly shaped unit cell is chosen. It is the parallelgram $A B C D$ shown in Fig. 7. From Eq. (3.9) and Fig. 6 one sees that for this $S_{8,8 ; 6,10}^{16}$ matrix element, where $l_{1}-l_{2}=-2$ and $j_{1}-j_{2}=2$,

$\pi k=\left(\Delta_{L}^{\prime}\right)_{B}-\left(\Delta_{L}^{\prime}\right)_{A}=\left(j_{1}-j_{2}\right) \pi$, whence $k=2$, $\pi K=\left(\Delta_{L}^{\prime}\right)_{D}-\left(\Delta_{L}^{\prime}\right)_{A}=\left(l_{1}-l_{2}\right) \pi+\left(j_{1}-j_{2}\right) \pi$, whence $K=0$.

\section{B. Two real and two complex stationary phase points}

To perform the calculation of the $S$ matrix for two real and two complex stationary phase points we apply

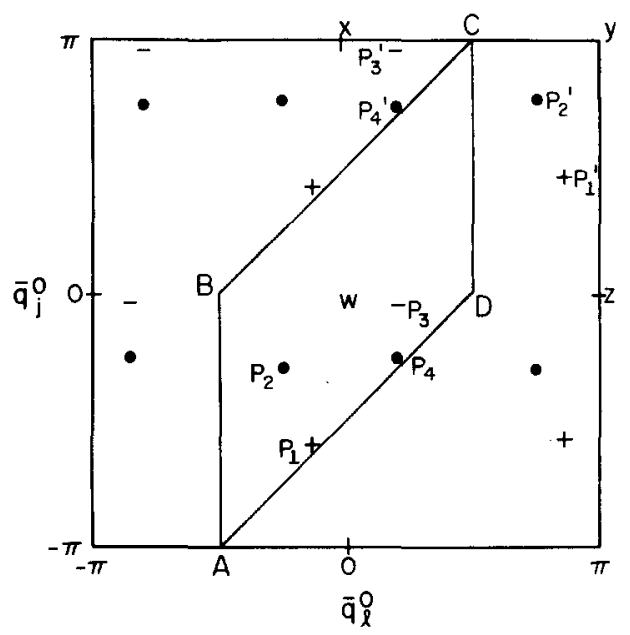

FIG. 7. Arrangement of stationary phase points in the space $\left(\bar{q}_{l}^{0}, \bar{q}_{j}^{0}\right)$ for the $S$ matrix $S_{8,8 ; 6,10^{\circ}}^{16}$ Maximum $(+)$, minimum (-), saddle points $(\bullet)$. The Bessel uniform approximation for the $S$ matrix can be used over the unit cell $A B C D$.

the criterion that the phases at the two real stationary phase points be as nearly equal as possible. The line connecting the two real points was treated an an " $A B$ " line and the value of $k$ was determined by using the element of the original unit cell most nearly parallel to the connecting line. This value of $k$ was used in Eqs. (B15) and (B17) in Appendix B.

\section{Summary}

The steps for performing the calculation of the $S$ matrix are summarized in the flow sheet in this section for the case of an atom plus homonuclear diatomic molecule, with a dominant $P_{2}(\cos \gamma)$ anisotropic term in the interaction potential (Appendix A). An atom and a heteronuclear diatomic molecule, with a dominant $P_{2}(\cos \gamma)$ anisotropy, will tend to have two or four stationary phase points in a $(-\pi, \pi)^{2}$ area. The same procedure would be followed with the following modifications: The $(0, \pi)^{2}$ in the flow sheet is replaced by $(-\pi, \pi)^{2}$. The $\pi^{2}$ in Step 2 is replaced by $(2 \pi)^{2}$. The $(-\pi, \pi)^{2}$ is replaced by $(-2 \pi, 2 \pi)^{2}$. Indeed in the latter, for transi-

TABLE II. Comparison of transition probabilities for the case of two real and two complex stationary phase points.

\begin{tabular}{|c|c|c|c|c|c|c|c|}
\hline \multirow[b]{2}{*}{$J$} & \multirow[b]{2}{*}{$l_{2}, j_{2} ; l_{1}, j_{1}$} & \multicolumn{4}{|c|}{ Bessel } & \multirow[b]{2}{*}{$\begin{array}{l}\text { Airy } \\
\text { Real } \\
\text { pts. }\end{array}$} & \multirow[b]{2}{*}{ Integral } \\
\hline & & Quantum ${ }^{a}$ & $\begin{array}{l}\text { Real } \\
\text { plus } \\
\text { complex pts. }\end{array}$ & $\begin{array}{l}\text { Real } \\
\text { pts. }\end{array}$ & $k^{\mathrm{b}}$ & & \\
\hline \multirow[t]{5}{*}{18} & 14,$6 ; 16,4$ & 0.124 & 0.103 & 0.069 & 4 & 0.069 & 0.125 \\
\hline & 8,$12 ; 10,10$ & 0.169 & 0.094 & 0.077 & 4 & 0.078 & 0.143 \\
\hline & 12,$10 ; 10,10$ & 0.087 & 0.057 & 0.059 & 2 & 0.057 & 0.099 \\
\hline & 10,$12 ; 10,10$ & 0.066 & 0.071 & 0.075 & 2 & 0.079 & 0.053 \\
\hline & 12,$8 ; 10,10$ & 0.165 & $\mathrm{c}$ & 0.085 & 4 & 0.086 & 0.163 \\
\hline \multirow[t]{2}{*}{6} & $8 ; 12,10$ & 0.201 & 0.195 & 0.133 & 4 & 0.134 & 0.203 \\
\hline & 6,$4 ; 4,4$ & 0.111 & 0.090 & 0.135 & 2 & 0.139 & $\mathrm{~d}$ \\
\hline
\end{tabular}

aReference 3.

${ }^{\mathrm{b}}$ Results were insensitive to either $k=2$ or 4 .

${ }^{c}$ We have not been able to locate the complex stationary phase points.

${ }^{d}$ The integral cannot be obtained due to Jacobian sign changes. 
Application of PSC and Uniform Approximations: Flow sheet I.
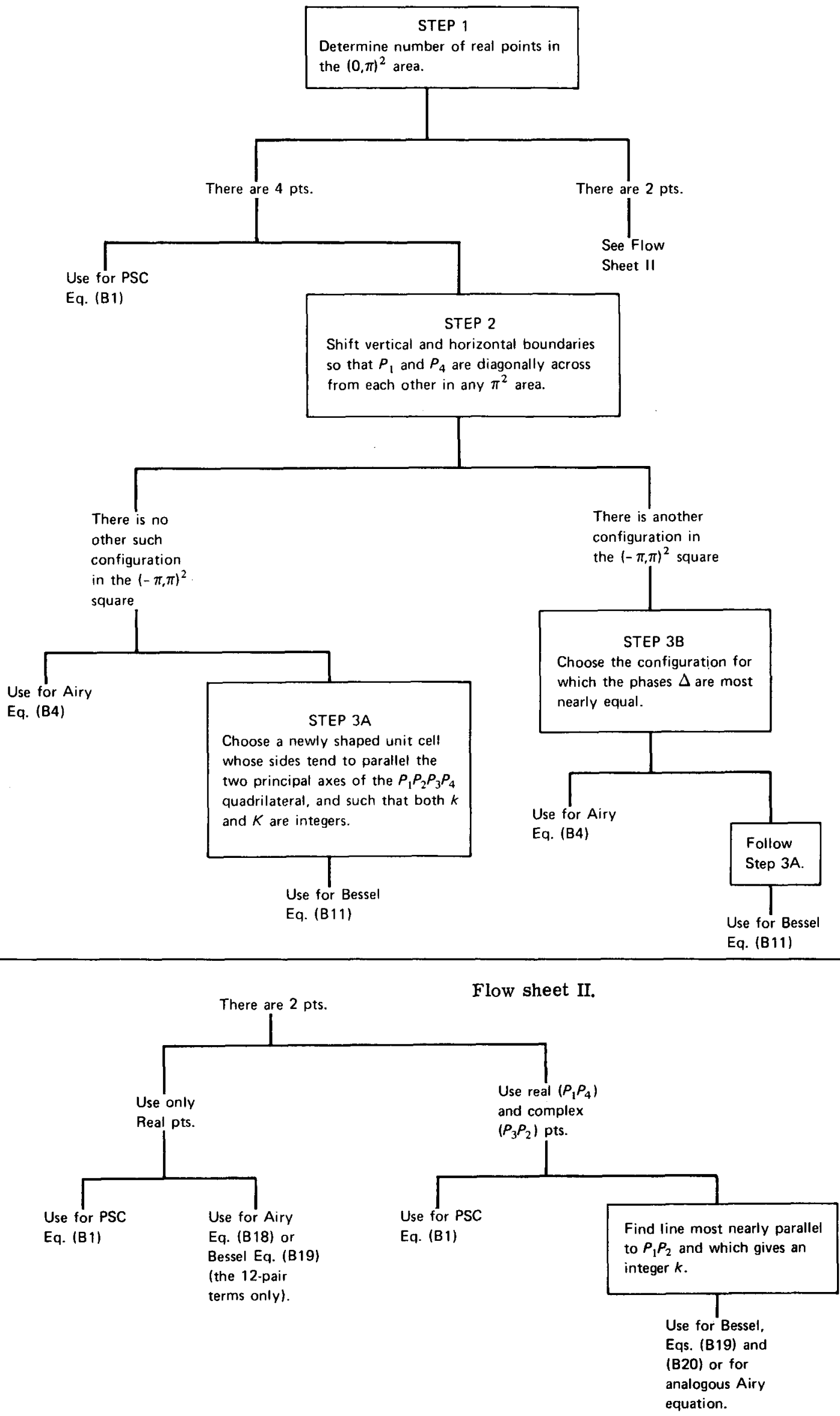
tions involving larger $\Delta l^{\prime}$ s and $\Delta j$ 's than those considered here, it may be necessary to scan a larger region than the $(-\pi, \pi)^{2}$ one for the homonuclear case or than the $(-2 \pi, 2 \pi)^{2}$ one for the heteronuclear case. The method described in Appendix $\mathrm{C}$ of first obtaining a contour plot for $\Delta$, after obtaining the stationary phase points, can be extremely useful for subsequently performing Steps 2 and 3 in the flow sheets for any new case.

When the interaction potential also contains large terms having $P_{n}(\cos \gamma)$ anisotropy with some of the $n$ 's being larger than 1 or 2 in these atom-linear molecule cases, D. Fitz of this laboratory has sometimes observed a larger number of stationary phase points.

\section{NUMERICAL RESULTS}

The results obtained for various transitions are given in Table I, where the Bessel and Airy uniform approximations are compared with the exact quantum ${ }_{3}$ results. ${ }^{3}$ The appropriate values of $K$ and $k$ obtained by the procedure in Sec. III are also indicated. Equations (B4) and (B11) of Appendix B were then applied. For all of the cases listed in Table I there were four real stationary phase points.

When two of the stationary phase points are real and the remainder are complex-valued, we use Eq. (B19) in complex form and Eq. (B20). As noted there, these equations can be less accurate than those used for Table I. The results obtained from these equations are given in Table II. If the terms arising from the complex-valued roots are omitted from these equations, one obtains the other results given in Table II. Comparison with the exact quantum results is also given.

It is useful to compare the exact quantum results given in the third column of Table I with approximate results less accurate than the Bessel uniform one: Airy uniform-in-pairs, Bessel uniform-in-pairs, classical, and primitive semiclassical. The relevant equations are given in Appendix B. There are two possible combinations of pairs for both uniform-in-pairs methods and both sets of results are listed in Table III.
When the correct configuration of stationary phase points is not used, e.g., if the maximum and minimum stationary phase points are not chosen diagonally across from each other, the results differ from those in Table I. For example, if one merely seeks the stationary phase points for which $0 \leq\left(\bar{q}_{l}^{0}, \bar{q}_{j}^{0}\right) \leq \pi$, as in Fig. 4, one obtains the results given in Table IV.

When the correct configuration of points is used for the Airy and the Bessel, but when a square unit cell is used for the latter instead of the cell favoring a better separation of variables, i.e., when the wrong $k$ and $K$ are used, one obtains the results given in Table V. In none of those results was $k$ or $K$ equal to zero, unlike the results in Table $\mathrm{I}$.

\section{DISCUSSION}

Agreement between the Bessel uniform approximation and the exact quantum results is excellent for transitions associated with four real stationary phase points in the $\pi^{2}$ area (Table I). As already noted, the Airy uniform is a special case of the Bessel ${ }^{16}$ but, as expected, is inferior to it in this case, in which $k$ or $K$ is zero (Table I). A similar behavior was found in Ref. 16 for the case of $r=1$, and is well understood: In that case the phase $\Delta$ varied only slowly and one could not, as one does in the Airy formalism, replace the limits $(-\pi, \pi)$ or $(0, \pi)]$ by $(-\infty,+\infty)$. Similarly, in the present two dimensional case, as in Fig. 8 in Appendix C, a plateau-like region for $\Delta$ occurs in one direction when $k$ or $K$ equals zero, and once again the Airy formalism breaks down.

In Table IV it is seen that the uniform-in-pairs approximation is not as good as the uniform formulas of Table I. The Bessel results indicate that the best choice of pairs is consistently the one which makes uniform the pair for which $k$ (or $K$ ) is zero. The reason for this behavior is clear, since in developing the pairs' equations given in Appendix B [Eqs. (B17) and (B19)] we assumed that one $\zeta$ was large. The $\zeta$ for the pair associated with $K$ or $k$ equal to zero was of the order of 0.16 , while the other pair had a $\zeta$ of the order of 2.5 in all cases studied. Thus, the former pair should give the better result in

TABLE II. Comparison of quantum transition probabilities with approximations less accurate than the Bessel: Four Real Stationary Phase Points in $\pi^{2}$ area. ${ }^{2}$

\begin{tabular}{|c|c|c|c|c|c|c|c|c|}
\hline \multirow[b]{2}{*}{$J$} & \multirow[b]{2}{*}{$l_{2}, j_{2} ; l_{1}, j_{1}$} & \multirow[b]{2}{*}{ Quantum } & \multicolumn{2}{|c|}{ Bessel in pairs } & \multirow{2}{*}{\multicolumn{2}{|c|}{ Airy in pairs }} & \multirow[b]{2}{*}{ Classical } & \multirow[b]{2}{*}{$\begin{array}{l}\text { Primitive } \\
\text { semiclassica }\end{array}$} \\
\hline & & & $\begin{array}{l}\text { Uniform for } \\
k=0 \text { or } K=0 \\
\text { Pair }\end{array}$ & $\begin{array}{l}\text { Uniform } \\
\text { for the } \\
\text { other pair }\end{array}$ & & & & \\
\hline \multirow[t]{4}{*}{16} & 12,$4 ; 10,6$ & 0.297 & 0.442 & 0.545 & 0.583 & 0.692 & 0.390 & 0.893 \\
\hline & 8,$8 ; 10,6$ & 0.296 & 0.417 & 0.678 & 0.616 & 0.734 & 0.449 & 0.755 \\
\hline & 4,$12 ; 6,10$ & 0.289 & 0.394 & 0.679 & 0.559 & 0.631 & 0.486 & 0.942 \\
\hline & 8,$8 ; 6,10$ & 0.285 & 0.402 & 0.721 & $0.633^{\circ}$ & 0.693 & 0.486 & 0.942 \\
\hline \multirow[t]{2}{*}{6} & $2, \quad 4 ; 4,2$ & 0.220 & 0.365 & 0.346 & 0.354 & 0.444 & 0.233 & 0.554 \\
\hline & 10,$4 ; 8,2$ & 0.297 & 0.444 & 0.778 & 0.771 & 0.797 & 0.605 & 1.189 \\
\hline \multirow[t]{2}{*}{14} & $12, \quad 2 ; 10$ & 0.275 & 0.386 & 0.518 & 0.516 & 0.528 & 0.849 & 1.893 \\
\hline & 8,$6 ; 10$ & 0.298 & 0.397 & c. 633 & 0.576 & 0.468 & 0.381 & 0.890 \\
\hline
\end{tabular}

aThe systems in this table are the same as in Table $I$.

${ }^{b}$ Pair A corresponds to the $(k=0$ or $K=0)$ pair in Bessel-in-pairs column. Pair $B$ corresponds to the other pairing. 
Table III, as it does, when it is treated as the pair which was made uniform.

The need for testing for a correct configuration of the stationary phase points, using the criterion established by the Hessian in Sec. III, is evident from Table IV, where the value for the uniform approximations obtained with the wrong configuration is seen to be quite different from the value obtained with the correct configuration, given in Table I. The only previous result with which to compare the results of Table $I$ is an intuitive Airy-type formula. ${ }^{4}$ In most of the instances in that work there were only two real-valued points, and the complex points were omitted. There were two cases where there were four points, and one is the same as the result in Table IV based on the wrong configuration. ${ }^{17}$

In previous papers of this series on multidimensional uniform approximations $s^{1,2}$ the Airy and Bessel uniform formulas were derived ${ }^{1,2,18,19}$ rather than intuitively interpolated, and so the assumptions and the concept of a correct configuration of points have become apparent.

It is useful to compare the Bessel and Airy uniform approximations for which only the square unit cell rather than a theoretically-based shaped one was used to determine $k$ and $K$. The results, given in Table $\mathrm{V}$ for the same systems as those in Table $I$, are clearly worse than the Bessel results in Table I. (The quantum results are given in Table $I_{0}$ ) The agreement of the Airy and Bessel uniform approximations in Table $\mathrm{V}$ with each other reflects the fact that for those systems both $k$ and $K$ are nonzero. The tendency of a Bessel uniform approximation to approach an Airy approximation provided the order of the Bessel function, $k$ or $K$, is not too small is evident from the relation of Bessel functions to Airy functions. ${ }^{19}$

Some systems having only two real stationary phase points in a $\pi^{2}$ area, given in Table $I$, are seen to have their approximate uniform values differ by a factor of about 2 or less from the exact quantum results when the complex-valued points are neglected. The agreement is improved, in some cases substantially, when the complex points are added and the complex pair formula used. However, overall the integral result remains superior for the particular data in Table $\Pi$.

The transition probabilities for the systems with two real points (Table II) are substantially lower than those

TABLE IV. Comparison of quantum transition probabilities with "Airy uniform" using incorrect configuration of stationary phase points. $^{\mathrm{a}}$

\begin{tabular}{lrlll}
\hline \hline$J$ & $l_{2}, j_{2} ; l_{1}, j_{1}$ & Quantum & "Airy" \\
\hline 16 & 12,$4 ; 10,6$ & 0.297 & 0.265 \\
& 8,$8 ; 10,6$ & 0.296 & 0.705 \\
& 4,$12 ; 6,10$ & 0.289 & 0.529 \\
& 8,$8 ; 10,6$ & 0.285 & 0.265 \\
6 & 2,$4 ; 4,2$ & 0.220 & 0.248 \\
& 10,$4 ; 8,2$ & 0.297 & 0.364 \\
& 14 & $2 ; 10,4$ & 0.275 & 0.142 \\
\hline \hline
\end{tabular}

${ }^{2}$ The systems in this table are the same as in Table I.
TABLE V. Comparison of Bessel and Airy transition probabilities using correct configuration beu with incorrect shape for unit cell for Bessel. Comparison of the "Bessel" and "Airy" approximation calculated from new but incorrect square unit cells. ${ }^{a}$

\begin{tabular}{|c|c|c|c|}
\hline \multirow[b]{2}{*}{$J$} & \multirow[b]{2}{*}{$l_{2}, j_{2} ; l_{1}, j_{1}$} & \multicolumn{2}{|c|}{ Probability } \\
\hline & & Airy & "Bessel",a \\
\hline 16 & 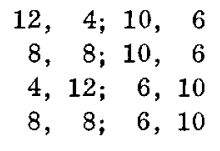 & $\begin{array}{l}0.451 \\
0.462 \\
0.378 \\
0.442\end{array}$ & $\begin{array}{l}0.436 \\
0.446 \\
0.365 \\
0.426\end{array}$ \\
\hline 6 & $\begin{array}{rrrr}2, & 4 ; & 4, & 2 \\
10, & 4 ; & 8, & 2\end{array}$ & $\begin{array}{l}0.285 \\
0.482\end{array}$ & $\begin{array}{l}0.276 \\
0.463\end{array}$ \\
\hline 14 & $\begin{array}{rrrr}12, & 2 ; & 10, & 4 \\
8, & 6 ; & 10, & 4\end{array}$ & $\begin{array}{l}0.382 \\
0.429\end{array}$ & $\begin{array}{l}0.370 \\
0.415\end{array}$ \\
\hline
\end{tabular}

${ }^{a}$ The systems in this table are the same as in Table I.

${ }^{\text {b The values of }}|K|$ and $|k|$ used in this (erroneous) Bessel approximation were each 2 .

for four real points (Table I), and one obtains the expected result ${ }^{16}$ that the Airy and Bessel uniform approximations yield similar answers in this instance. The integral results from an earlier pape ${ }^{10}$ for this case are also noted in Table II, and they are in very good agreement with the quantum results.

\section{CONCLUSIONS}

In summary, the results in Table I show that good agreement of exact and semiclassical results is obtained for the case of high transition probabilities (four stationary phase points in the present instance). The reason for the improvement of the Bessel over the Airy for any case where a $k$ or $K$ is zero is evident from an examination of Fig. 8. The need for choosing a correct configuration and a correctly shaped unit cell is also clear.

The agreement for the case where two of the points are complex (Table II) is reasonable though not quite as good, probably because of the extra approximation of using a uniform-in-pairs formula.

\section{APPENDIX A: THE ATOM-RIGID ROTOR COORDINATES AND HAMILTONIAN}

The classical Hamiltonian for the atom rigid-rotor system is the well known generalized coordinate system used in previous studies. ${ }^{4,9,20}$ In the present work we will use a Lennard-Jones interaction potential of the form used in several recent "exact" quantum dynamical studies. ${ }^{3}$ The total Hamiltonian in the center of mass system is

$H=\frac{P_{r}^{2}}{2 \mu}+\frac{\alpha_{1}^{2}}{2 \mu r^{2}}+\frac{\alpha_{2}^{2}}{2 I}+\epsilon\left[\left(\frac{r_{m}}{r}\right)^{12}-2\left(\frac{r_{m}}{r}\right)^{6}\right]\left[1+a P_{2}(\cos \gamma)\right]$.

$P_{r}$ is the momentum of relative motion of the atom and center of mass of the rotor and $r$ is the conjugate coordinate. $\alpha_{1}$ and $\alpha_{2}$ are the angular momentum of the orbital and rotational motions, respectively. $I$ is the moment of inertia of the rotor, $\epsilon$ and $r_{m}$ are the welldepth and the position of the minimum of the interaction potential. $a$ is an asymmetry parameter of the potential. 
$\gamma$ is the angle between the axis of the rotor and the line joining the center of mass of the rotor and the atom:

$\cos \gamma=-\cos q_{1} \cos q_{2}+\left[\alpha_{1}^{2}+\alpha_{2}^{2}-\alpha_{3}^{2} / 2 \alpha_{1} \alpha_{2}\right] \sin q_{1} \sin q_{2}$,

where $\alpha_{3}$ is the total angular momentum of the system and the coordinates $q_{1}$ and $q_{2}$ are conjugate to $\alpha_{1}$ and $\alpha_{2}{ }^{20}$

It is convenient to employ the Hamiltonian (A1) in reduced units. The reduced Hamiltonian $H^{1}$ is

$H^{\prime}=H / \epsilon=\frac{P_{R}^{2}}{2}+\frac{\hat{l}^{2}}{2 R^{2}}+\frac{\hat{j}^{2}}{2 I^{\prime}}+\left(R^{-12}-2 R^{-6}\right)\left[1+a P_{2}(\cos \gamma)\right]$,

where $P_{R}, R, q_{l}, q_{j}, \hat{l}, \hat{j}, I^{\prime}$, and $\hat{J}$ are the dimensionless quantities $P_{r} / \sqrt{\mu \epsilon}, r / r_{m}, q_{1}, q_{2}, \alpha_{1} /\left(r_{m} \sqrt{\mu \epsilon}\right), \alpha_{2} /$ $\left(r_{m} \sqrt{\mu \epsilon}\right), I / \mu r_{m}^{2}$ and $\alpha_{3} /\left(r_{m} \sqrt{\mu \epsilon}\right)$, respectively. The expression for cos $\gamma$ becomes

$\cos \gamma=-\cos q_{l} \cos q_{j}+\left[\left(\hat{l}^{2}+\hat{j}^{2}-\hat{J}^{2}\right) / 2 \hat{l} \hat{j}\right] \sin q_{l} \sin q_{j}$.

In these reduced units the instantaneous values of the angular momenta are related to the values of the "quantum numbers" by the semiclassical relation,

$$
\hat{l}=\left(l+\frac{1}{2}\right) \hbar_{0}, \quad \hat{j}=\left(j+\frac{1}{2}\right) \hbar_{0}, \quad \hat{J}=\left(J+\frac{1}{2}\right) \hbar_{0},
$$

where $\hbar_{0}$ is $\hbar /\left(r_{m} \sqrt{\mu \epsilon}\right)$. In the present study the following set of parameters was used: $I^{\prime}=0.4, a=0.25$, and $\left[\hbar /\left(r_{m} \sqrt{\mu \epsilon}\right)\right]^{2}=0.002$; in all cases the total energy of the system was $E=1$.

Initial conditions for the trajectory calculations are determined by specifying the total energy $E$ of the system, the initial values of the quantum numbers $l, j$, and $J$, and the angles $q_{l}$ and $q_{j}$ for some given initial large value of $R$. Following previous notation, the initial values of $l, j$, and $J$ are the integers $l_{1}, j_{1}$, and $J_{1}$ and the initial values of $q_{l}$ and $q_{j}$ are $q_{l}^{0}$ and $q_{j}^{0}$. The initial values of $\hat{l}, \hat{j}, \hat{J}, R$, and $P_{R}$ are denoted by $\hat{l}_{1}, \hat{j}_{1}, \hat{J}_{1}, R_{1}$, and $P_{R_{1}}$. We used the new variables $\bar{q}_{l}^{0}$ and $\bar{q}_{j}^{0}$ defined on the interval $-\pi$ to $\pi$. These values are related to $q_{1}^{0}$ and $q_{j}^{0}$ by $^{10,21}$

$$
\bar{q}_{l}^{0}=q_{l}^{0}-\tan ^{-1}\left(P_{R 1} R_{1} / \hat{l}_{1}\right)-\frac{1}{2} \pi,
$$

where in (A6) and (A8) the $\tan ^{-1}$ 's lie in $\left(-\frac{1}{2} \pi, 0\right)$ and $(0$, $\left.\frac{1}{2} \pi\right)$ intervals, respectively. The $\frac{1}{2} \pi^{\prime} s$ are omitted throughout the text and the figures. (They do not affect $\left|S_{m n}\right|^{2}$.) Also,

$$
\bar{q}_{j}^{0}=q_{j}^{0}-\nu_{j}^{0} \mu R_{1} P_{R_{1}} /\left[P_{R_{1}}^{2}+\left(\hat{l}^{2} / R_{1}^{2}\right)\right],
$$

where

$$
\nu_{f}^{0}=\hat{j}_{1} / I^{\prime} \text {. }
$$

Continuing to follow previous notation, the final values of the momentum and coordinates for any trajectory are $\hat{l}^{f}, \hat{j}^{f}, \hat{J}^{f}, q_{l}^{f}, q_{j}^{f}, R^{f}, P_{R}^{f}, \bar{q}_{l}^{f}$, and $\bar{q}_{j}^{f}$, where

$$
\begin{aligned}
& \bar{q}_{l}^{f}=q_{l}^{f}-\tan ^{-1}\left(P_{R 2} R_{2} / \hat{l}_{2}\right)+\frac{1}{2} \pi, \\
& \nu_{l}^{f}=\hat{l}^{f} /\left(R^{f}\right)^{2}, \\
& \bar{q}_{j}^{f}=q_{j}^{f}-\nu_{j}^{f} \mu R^{f} P_{R}^{f} /\left\{\left(P_{R}^{f}\right)^{2}+\left[\left(\hat{l}^{f}\right)^{2} /\left(R^{f}\right)^{2}\right]\right\}, \\
& \nu_{j}^{f}=\hat{j}^{f} / I^{\prime} .
\end{aligned}
$$

\section{APPENDIX B: NONUNIFORM AND UNIFORM EXPRESSIONS FOR THE $S$-MATRIX}

\section{Primitive semiclassical and classical-like results}

The primitive semiclassical result for the $S$ matrix, $S_{\mathrm{PSC}}$, and for the transition probability, $P_{\mathrm{PSC}}$, calculated from the sixteen stationary phase points in the $(2 \pi)^{2}$ domain, are obtained from

$$
S_{\mathrm{PSC}}=4 \sum_{i=1}^{4} p_{i} e^{i f_{i}}, \quad P_{\mathrm{PSC}}=\left|S_{\mathrm{PSC}}\right|^{2}
$$

where $p_{i}$ is a signed determinant ${ }^{18,19,22}$ :

$$
p_{i}=\frac{1}{2 \pi}\left|\frac{\partial\left(l^{f}, j^{f}\right)}{\partial\left(\bar{q}_{l}^{0}, \bar{q}_{j}^{0}\right)}\right|_{i}^{-1 / 2},
$$

and $i=1-4$ denote the four stationary phase points in a $\pi^{2}$ domain; $f_{i}$ is the value of $\Delta$ in Eq. (3.3) at the stationary phase point $P_{i}$.

When $G$ is positive at a stationary phase point $P_{i}$, as in the case of the results given in Table $I$, the sign of $\left|\partial\left(l^{f}, j^{f}\right) / \partial\left(\bar{q}_{l}^{0}, \bar{q}_{j}^{0}\right)\right|$ at that $P_{i}$ in Eq. (B2) is the same as that of $\left.\mid \partial\left(l^{f}, j^{f}\right) / \partial\left(\bar{q}_{l}, \bar{q}_{j}\right)\right)$, since $\left|\partial\left(l^{f}, j^{f}\right) / \partial\left(\bar{q}_{l}^{f}, \bar{q}_{j}^{f}\right)\right| G$ equals $\left|\partial\left(l^{f}, j^{f}\right) / \partial\left(\bar{q}_{l}^{0}, \bar{q}_{j}^{0}\right)\right|$. When the phase $\Delta$ is a maximum, one can show that $\left|\partial\left(l^{f}, j^{f}\right) / \partial\left(\bar{q}_{j}^{f}, \bar{q}_{l}^{f}\right)\right|$ is $-2 \pi i$, while for a saddle point it is $-\pi i$, and for a minimum it is 0 . Thus, the phases of $p_{i}$ in Eq. (B2) for these respective cases are $\pi i, \frac{1}{2} \pi i$, and 0 , for this case of positive $G$.

A more classical-like result for the transition probability $p_{c}$ is obtained from Eq. (B1) by neglecting interference terms and is

$$
p_{c}=16 \sum_{i=1}^{4} p_{i}^{2}
$$

when all four stationary phase points are real; if any of the four points are complex-valued, the corresponding $p_{i}$ 's are ignored in Eq. (B3).

\section{Airy uniform approximation}

We have developed two uniform approximations for evaluating the semiclassical $S$ matrix. They are the Airy and Bessel uniform approximations. ${ }^{1,2}$ The Airy approximation yields $S_{\text {Atry }}$ for the $S$ matrix:

$$
\begin{aligned}
S_{\text {A } 1 Y y}= & 2 i\left[G_{1}^{-1 / 2} a i^{+}\left(-\eta_{12}\right) a i^{+}\left(-\eta_{14}\right)\left(\eta_{14} \eta_{12}\right)^{1 / 4} e^{i A_{1}+i \pi / 2}\right. \\
& +G_{2}^{-1 / 2} a i^{-}\left(-\eta_{12}\right) a i^{+}\left(-\eta_{23}\right)\left(\eta_{12} \eta_{23}\right)^{1 / 4} e^{i A_{2}} \\
& +G_{3}^{-1 / 2} a i^{-}\left(\eta_{23}\right) a i^{-}\left(-\eta_{43}\right)\left(\eta_{23} \eta_{43}\right)^{1 / 4} e^{i A_{3}-i \pi / 2} \\
& +G_{4}^{-1 / 2} a i^{-}\left(-\eta_{14}\right) a i^{+}\left(-\eta_{43}\right)\left(\eta_{14} \eta_{43}\right)^{1 / 4} e^{\left.i A_{4}\right]}
\end{aligned}
$$

where $G_{i}$ is the value of $G$ in Eq. (3.2) at the point $P_{i}$, $A_{2}$ is given by

$$
A_{2}=\frac{1}{2}\left(f_{1}+f_{3}\right) \text {, }
$$

and the remaining $A_{i}$ 's are obtained by cyclic permutations of the indices

$$
\begin{array}{ll}
\frac{4}{3} \eta_{14}^{3 / 2}=f_{4}-f_{1}, & \frac{4}{3} \eta_{12}^{3 / 2}=f_{2}-f_{1}, \\
\frac{4}{3} \eta_{23}^{3 / 2}=f_{3}-f_{2}, & \frac{4}{3} \eta_{43}^{3 / 2}=f_{3}-f_{4} .
\end{array}
$$

Furthermore,

$$
a i^{ \pm}(-\eta)=A i(-\eta) \pm i \eta^{-1 / 2} A i^{\prime}(-\eta)
$$




$$
A i(-\eta)=(2 \pi)^{-1} \int_{-\infty}^{\infty} \exp i\left(\frac{1}{3} u^{3}-\eta u\right) d u
$$

and prime $\left({ }^{\prime}\right)$ denotes derivation of $A i(-\eta)$ with respect to $-\eta$.

\section{Bessel uniform approximation}

As discussed in the preceding paper, ${ }^{1,23}$ along a $u$ curve we have

$$
\Delta\left(\bar{q}_{i}^{0}, \bar{q}_{j}^{0}\right) \equiv \bar{f}(u, v)=K u-\zeta \sin N u+A
$$

and along a $v$-curve we have

$$
\Delta\left(\bar{q}_{l}^{0}, \bar{q}_{j}^{0}\right) \equiv \bar{f}(u, v)=k v-\zeta \sin M v+A,
$$

where the $A$ and $\zeta$ in $\mathrm{Eq}$. (B9) differ in value from those in $\mathrm{Eq} .(\mathrm{B} 10)$. $N$ and $M$ denote the periodicities, i. e. , $\Delta$ contains a periodic function of $u$ and $v$ with a period of $2 \pi / N$ and $2 \pi / M$, respectively.

$S_{\text {Besse 1 }}$ is the Bessel uniform approximation for the $S$ matrix $^{24}$ :

$$
\begin{aligned}
& S_{\text {Besse } 1}=\frac{1}{4} N M\left[\left(G_{1}^{-1} \zeta_{14} \zeta_{12} \sin \bar{u}_{1} \sin \bar{v}_{1}\right)^{1 / 2} \mathcal{J}_{\bar{K}}^{+}\left(\zeta_{14}\right) \mathcal{J}_{\bar{k}}^{+}\left(\zeta_{12}\right) e^{i A_{1}}+\left(G_{2}^{-1} \zeta_{23} \zeta_{12} \sin \bar{u}_{2} \sin \bar{v}_{2}\right)^{1 / 2} g_{\bar{K}}^{+}\left(\zeta_{23}\right) \mathcal{J}_{\bar{k}}^{-}\left(\zeta_{12}\right) e^{i A_{2}}\right.
\end{aligned}
$$

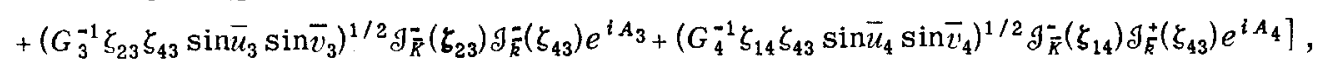

where $A_{2}$ is given by (B5), the other $A_{i}$ 's are given by cyclic permutation of the indices, and

$$
\bar{u}_{i} \equiv N u_{i}, \quad \overline{v_{i}} \equiv M v_{i}, \quad \bar{K}=K / N, \quad \bar{k}=k / M .
$$

The $\bar{u}_{i}$ and $\bar{v}_{i}$ in (B11) are related to the value of the phase at the stationary phase points:

$$
\left.\begin{array}{ll}
\bar{K}\left(\bar{u}_{4}-\tan \bar{u}_{4}\right)=\frac{1}{2}\left(f_{4}-f_{1}\right) ; \bar{u}_{1}=-\bar{u}_{4} \\
\bar{K}\left(\bar{u}_{3}-\tan \bar{u}_{3}\right)=\frac{1}{2}\left(f_{3}-f_{2}\right) ; \bar{u}_{2}=-\bar{u}_{3} \\
\zeta_{14}=\bar{K} / \cos \bar{u}_{4}, \quad \zeta_{23}=\bar{K} / \cos \bar{u}_{3} \\
\bar{k}\left(\bar{v}_{2}-\tan \bar{v}_{2}\right)=\frac{1}{2}\left(f_{2}-f_{1}\right) ; \bar{v}_{1}=-\bar{v}_{2} \\
\bar{k}\left(\bar{v}_{3}-\tan \bar{v}_{3}\right)=\frac{1}{2}\left(f_{3}-f_{4}\right) ; \bar{v}_{4}=-\bar{v}_{3} \\
\zeta_{12}=\bar{k} / \cos \bar{v}_{2}, \quad \zeta_{43}=\bar{k} / \cos \bar{v}_{3} .
\end{array}\right\}
$$

The first two equations in (B12a) are solved for the $\bar{u}_{i}$ 's. The third equation is then solved for the $\zeta_{i j}$ 's. Similar remarks apply to the three equations in (B12b). We also have

$$
\left.\begin{array}{ll}
\bar{u}_{1}=\bar{u}_{2}=-\pi / 2, & \bar{u}_{4}=-\bar{u}_{1}, \bar{u}_{3}=-\bar{u}_{2} \\
\zeta_{12}=\frac{1}{2}\left(f_{2}-f_{1}\right), & \zeta_{43}=\frac{1}{2}\left(f_{3}-f_{4}\right) \quad
\end{array}\right\}(\bar{K}=0)
$$

In the cases $\bar{K} \neq 0, \bar{k}=0$ or $\bar{K}=0, \bar{k} \neq 0$, the appropriate combination in Eqs. (B12) is used.
The function $d$ in Eq. (B11) is given in terms of Bessel functions of integer order:

$$
\begin{aligned}
& g_{R}^{ \pm}\left(\zeta_{i j}\right)=J_{\left|R^{\prime}\right|}\left(\left|\zeta_{i j}\right|\right) \pm i J_{\left|R^{\prime}\right|}^{\prime}\left(\left|\zeta_{i j}\right|\right)\left[\left|\sin u_{j}\right|\right], \text { if } i j=14 \text { or } 23 \\
& g_{\bar{k}}^{ \pm}\left(\zeta_{i j}\right)=J_{|\bar{k}|}\left(\left|\zeta_{i j}\right|\right) \pm i J_{|\bar{k}|}^{\prime}\left(\left|\zeta_{i j}\right|\right)\left[\left|\sin v_{f}\right|\right], \text { if } i j=12 \text { or } 43 .
\end{aligned}
$$

The symmetry properties of Bessel functions of real argument were used in obtaining the rhs of these equations. ${ }^{24}$

\section{Uniform-in-pairs approximation}

Another possible expression for the $S$ matrix can be written if the stationary phase points are considered to occur in pairs which are "well-separated". By wellseparated we mean that the relevant $\eta$ 's and $\zeta$ 's are large. If the (12) and (43) pair are well-separated the values of $\eta_{14}$ and $\eta_{23}$ are large and Eq. (B4) for the Airy approximation gives

$S_{\mathrm{A} 1 \mathrm{yy}}=B i^{+}\left(-\eta_{12}\right) I_{1}+B i^{-}\left(-\eta_{12}\right) I_{2}+B i^{-}\left(-\eta_{43}\right) I_{3}+B i^{+}\left(-\eta_{43}\right) I_{4}$.

The $B i$ 's are given by Eq. (19c) of Ref. 2. If the Bessel uniform approximations $\zeta_{14}$ and $\zeta_{23}$ become large, then Eq. (B11) gives

$$
\begin{aligned}
S_{\mathrm{Besse}}= & (2 / \pi)^{1 / 2} \frac{1}{4} N M\left\{\exp \left[i\left(f_{1}+f_{2}\right)-\frac{1}{4} i \pi\right]\left[\left(G_{1}^{-1} \zeta_{12} \sin \bar{v}_{1}\right)^{1 / 2} J_{\bar{k}}^{+}\left(\zeta_{12}\right)+\left(G_{2}^{-1} \zeta_{12} \sin \bar{v}_{2}\right)^{1 / 2} \mathrm{~J}_{\bar{k}}\left(\zeta_{12}\right)\right]\right. \\
& \left.+(2 / \pi)^{1 / 2} \exp \left[i\left(f_{3}+f_{4}\right)+\frac{1}{4} i \pi\right]\left[\left(G_{3}^{-1} \zeta_{43} \sin \bar{v}_{3}\right)^{1 / 2} g_{\bar{k}}\left(\zeta_{43}\right)+\left(G_{4}^{-1} \zeta_{43} \sin \bar{v}_{4}\right)^{1 / 2} g_{\bar{k}}^{+}\left(\zeta_{43}\right)\right]\right\} .
\end{aligned}
$$

If the (14) and (23) pairs are well-separated, $\eta_{12}$ and $\eta_{43}$ will be large in the Airy case while $\zeta_{12}$ and $\zeta_{43}$ will be large in the Bessel case. The Airy and Bessel approximation for this case are the following:

$$
\begin{aligned}
S_{\mathrm{A} 1 \mathrm{ry}}= & B i^{+}\left(-\eta_{14}\right) I_{1}+B i^{+}\left(-\eta_{23}\right) I_{2}+B i^{-}\left(-\eta_{23}\right) I_{2}+B i^{-}\left(\eta_{14}\right) I_{4}, \\
S_{\mathrm{B} \theta \mathrm{ss \theta} 1}= & (2 / \pi)^{1 / 2} \frac{1}{4} N M\left\{\exp \left[i\left(f_{1}+f_{4}\right)-\frac{1}{4} i \pi\right]\left[\left(G_{1}^{-1} \zeta_{14} \sin \bar{u}_{1}\right)^{1 / 2} g_{\bar{R}}^{+}\left(\zeta_{14}\right)+\left(G_{2}^{-1} \zeta_{23} \sin \bar{u}_{2}\right)^{1 / 2} \mathrm{~J}_{\bar{K}}^{+}(23)\right]\right. \\
& \left.+(2 / \pi)^{1 / 2} \exp \left[i\left(f_{2}+f_{3}\right)+\frac{1}{4} i \pi\right]\left[\left(G_{3}^{-1} \zeta_{23} \sin \bar{u}_{3}\right)^{1 / 2} g \overline{\tilde{K}}^{\prime}\left(\zeta_{23}\right)+\left(G_{4}^{-1} \zeta_{14} \sin \bar{u}_{4}\right)^{1 / 2} g_{\bar{K}}\left(\zeta_{14}\right)\right]\right\} .
\end{aligned}
$$

\section{Two real and two complex points: uniform-in-pairs approximation}

Finally, if two of the stationary phases are real and two are complex, let $P_{3}$ and $P_{4}$ denote the complex-val- ued points. Let $P_{1}$ be the maximum and $P_{2}$ the minimum in the real-valued pair. One may write an expression for the $S$ matrix in the Bessel approximation of the same form as Eq. (B15); but now, $f_{3}, f_{4}, G_{3}, G_{4}, v_{3}$, 
and $v_{4}$ are complex quantities. From the treatment of this problem given in the previous paper, ${ }^{1,25} v_{3}$ and $v_{4}$ are determined from

$$
i \bar{k}\left(\bar{v}_{3}^{\prime \prime}-\tanh \bar{v}_{3}^{\prime \prime}\right)=\frac{1}{2}\left(f_{3}-f_{4}\right), \quad \bar{v}_{4}^{\prime \prime}=-\bar{v}_{3}^{\prime \prime},
$$

where $\bar{v}^{\prime \prime}=\operatorname{Im}(\bar{v})$, and $\operatorname{Re}(\bar{v})=0 . \quad \zeta_{43}$ is given by

$$
\zeta_{43}=\bar{k} / \sinh v_{3}^{\prime \prime} \text {. }
$$

The $k$ for the real-valued pair is determined from $\mathrm{Eq}$. (B15). In the complex-valued pair its Bessel uniform approximation (the $\zeta_{43}$ terms) would approach an Airy one and so its $k$ would be immaterial, as long as $k \neq 0$. When using $\mathrm{Eq}$. (B15) the $k$ for the real pair was simply used. One can also simply replace the Bessel formula for the complex pair by the corresponding Airy one.

\section{APPENDIX C: DETAILED EXAMINATION OF A PHASE CONTOUR PLOT}

Insight into the procedure in the flow sheet and into conditions where the Bessel formalism yields a different (more correct) result than the Airy one is obtained from the examination of a plot of contours of constant phase of $\Delta\left(\bar{q}_{l}^{0}, \bar{q}_{j}^{0}\right)$. Such a plot is given in Fig. 8 for the case of a determination of $S_{4,12 ; 6,10}^{16}$. This is the same example used for Figs. 4 and 6 . The contours were obtained by a program which interpolates smoothly the values of the phase $\Delta\left(\bar{q}_{l}^{0}, \bar{q}_{j}^{0}\right)$ on a $10 \times 10 \mathrm{grid}$ of points. ${ }^{8}$ The contours in Fig. 8 are equispaced with an interval of 0.2094 . The $10 \times 10$ grid proved to be too coarse to allow the program to properly interpolate in the region of saddle points. Consequently we have handsketched the contours in these regions.

Where the contours in Fig. 8 are densely spaced, $\Delta$ is rapidly changing, whereas where they are widely spaced $\Delta$ is only slowly varying. One sees, therefore,

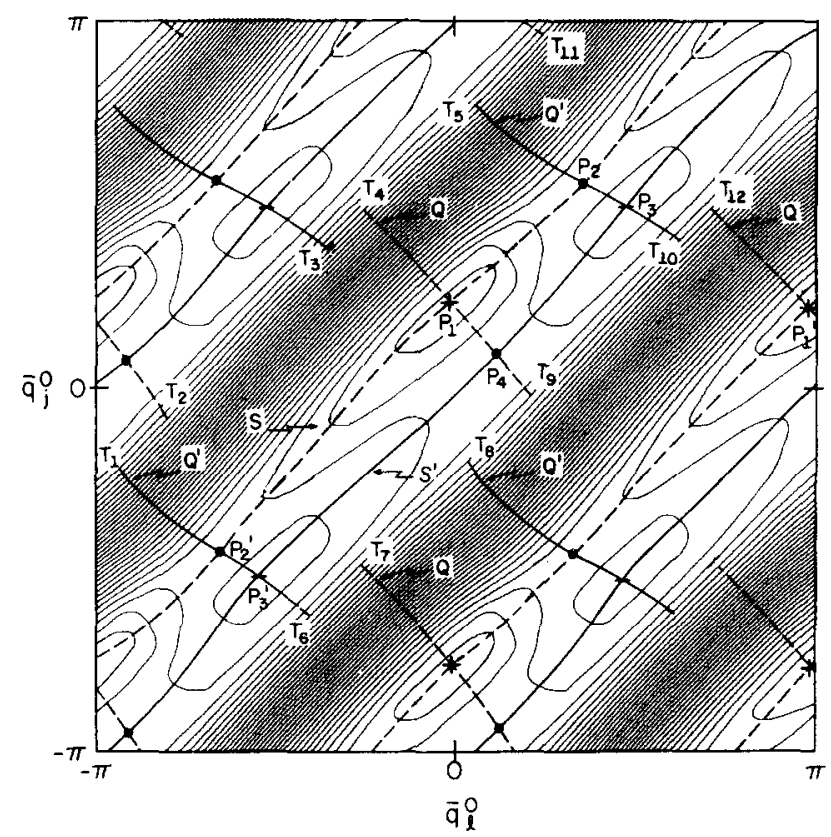

FIG. 8. Contours of constant phase $\Delta$ in the space $\left(\bar{q}_{l}^{0}, \bar{q}_{j}^{0}\right)$ for the $S$ matrix $S_{4,12 ; 6,10}^{16}$. Maximum $(+)$, minimum $(-)$, saddle point $(\bullet)$, curves of local transverse maxima $(--)$, curves of local transverse minima (- $)$. that there are largely diagonally inclined plateau regions where $\Delta$ is only slowly varying. For this reason, and indeed as in a corresponding one-dimensional case, ${ }^{16}$ the Airy formalism is not very accurate for such a system.

To see how a newly shaped curvilinear unit cell can be selected for use in a Bessel formalism and to make concrete the $u$ - and $v$-curves referred to in the preceding papers ${ }^{1,2}$ and in Appendix B it is useful to draw the curves of steepest descent and ascent from the maximum, minimum and saddle-points, as is done schematically in Fig. 8.

These curves prove to be the most important $u$ - and $v$-curves in the preceding papers, ${ }^{1,2}$ namely those passing through the stationary phase points: The $u$-curve passing through the maximum $P_{1}$ and a saddle-point $P_{4}$ is the locus of a set of points which are local maxima on curves transverse to $P_{1} P_{4}$ and so is a path of steepest descent from $P_{1}$. We call it a $Q$-curve. Similar remarks apply to the $v$-curve passing through $P_{1}$ and $P_{2}$ and we call it an $S$-curve. The $S$ - and $Q$-curves are dashed lines in Fig. 8 . The $u$-curve passing through the minimum $P_{3}$ and saddle point $P_{2}$ is a path of steepest ascent from $P_{3}$, being the locus of a set of points which are local minima along the curves transverse to $P_{3} P_{2}$. We call it a $Q^{\prime}$-curve. Similar remarks apply to the $v$-curves passing through $P_{3}$ and $P_{4}$, and we call it an $S^{\prime}$-curve and $Q^{\prime}$-curves in Fig. 8 are solid curves passint through the $P$ 's. In the periodic array in Fig. 8, the primed $P_{i}^{\prime}$ s are the same as the $P_{i}^{\prime}$ 's but displaced by the appropriate periods.

An interesting feature of the contour plot in Fig. 8 is the abrupt termination of the $Q^{\prime}$-curve $T_{1} P_{2}^{\prime} P_{3}^{\prime} T_{6}$ at the points $T_{1}$ and $T_{6}$. Along this solid $Q^{\prime}$ curve, which is a locus of the minima of curves transverse to $P_{3} P_{2}$, the derivative in the direction transverse to the curve equals zero and so is also equal to zero at the points $T_{1}$ and $T_{6}$. Beyond $T_{1}$ and $T_{6}$ it would continue as a locus of local maxima instead of local minima. Similarly, the Qcurve $T_{4} P_{2} P_{4} T_{9}$ terminates at $T_{4}$ and $T_{9}$. Beyond those points it would continue as a locus of local minima instead of local maxima.

The loci of all such termination points, e.g., $T_{1} T_{2} T_{3} T_{4} T_{5}$ and $T_{6} T_{7} T_{8} T_{9} T_{10}$ in Fig. 8, from two natural boundaries to the true unit cell on which to base a derivation of the uniform approximation.

To corretcly apply the curvilinear mapping of $\Delta\left(\bar{q}_{l}^{0}\right.$, $\left.\bar{q}_{j}^{0}\right)$ onto a function $\bar{f}(u, v)$ in Eq. (B9) and (B10), one should first select a suitable unit cell of area $\pi^{2}$, with. the property that the $Q$ and $Q^{\prime}$ curves terminate on the boundary of the unit cell. A suitable unit cell is in Fig. 8 and is enclosed by the four curves $T_{1} T_{2} T_{3} T_{4} T_{5}, T_{1} T_{6}$, $T_{6} T_{7} T_{8} T_{9} T_{10}$, and $T_{5} T_{10}$. Each point in the new unit cell corresponds to a point in the old square unit cell, with corresponding points having the same value of $G$ and the same value of $\exp i \Delta$, and so the integral in Eq. (3.1) evaluated over the new cell equals the integral evaluated over the old.

In Fig. 8 two of the four stationary phase points (s.p. points) lie on the boundary of the $T_{1} T_{5} T_{10} T_{6}$ unit cell. 
In order that all four s.p. points lie inside a unit cell, it suffices, in principle, to find the $u$-curve passing through $\left(T_{2}, T_{7}\right)$ and the one passing through $\left(T_{11}, T_{12}\right)$. The new unit cell $T_{2} T_{11} T_{12} T_{7}$ new enclosed the four $s . p$. points $P_{1}, P_{2}, P_{3}$, and $P_{4}$. This construction of a new unit cell leaves the value of the $S$ matrix unchanged, since each point in the newly constructed unit cell is equivalent to a point in the old unit cell $0 \leq \bar{q}_{l}^{0} \leq \pi$, $0 \leq \bar{q}_{j}^{0} \leq \pi$. For the purpose of the following discussion we shall consider the points $T_{1}, T_{5}, T_{10}$, and $T_{6}$ to refer in general to the corners of any one of the unit cells which can be constructed so as to enclose the s.p. points

Since the curve $T_{1} T_{6}$ is a $u$-curve we may use Eq. (B9) for $\bar{f}(u, v)$ and use the relation between the value of $u$ at $T_{1}$ and $T_{B}$,

$$
u\left(T_{6}\right)=u\left(T_{1}\right)+\pi,
$$

to determine the value of $K$ to be used in the mapping. We find from (B9) that

$$
\pi K=\left[\Delta\left(\bar{q}_{l}^{0}, \bar{q}_{j}^{0}\right)\right]_{T_{6}}-\left[\Delta\left(\bar{q}_{l}^{0}, \bar{q}_{j}^{0}\right)\right]_{T_{1}} .
$$

The same value of $K$ would be obtained from any other similar pair of transition points in this array (i.e. , the pairs $T_{4} T_{9}$ and $\left.T_{5} T_{10}\right)$.

To determine the value of $k$, we can use the fact that $T_{1}$ and $T_{5}$ lie on the same $v$-curve and are related by

$$
v\left(T_{5}\right)=v\left(T_{1}\right)+\pi \text {. }
$$

The value of $k$ obtained using Eq. (B10) is

$$
\pi k=\left[\Delta\left(\bar{q}_{l}^{0}, \bar{q}_{j}^{0}\right)\right]_{T_{5}}-\left[\Delta\left(\bar{q}_{l}^{0}, \bar{q}_{j}^{0}\right)\right]_{T_{1}} \text {. }
$$

To avoid the necessity of knowing the phase at points other than the stationary phase points in order to apply the Bessel uniform approximation, we can construct an approximate unit cell in the form of a parallelogram through the corners of the unit cell $T_{1} T_{5} T_{10} T_{6}$. Then the linear terms of the phase in $(u, v)$ space given by $\mathrm{Eq}$. (C2) and (C4) may be equated to the linear terms of the phase given by Eq. (3.6). ${ }^{28}$

$$
\begin{aligned}
& \text { Thus, } \\
& \pi k=\left(\Delta_{L}^{\prime}\right)_{T_{8}}-\left(\Delta_{L}^{\prime}\right)_{T 1}, \quad \pi k=\left(\Delta_{L}^{\prime}\right)_{T_{5}}-\left(\Delta_{L}^{\prime}\right)_{T 1}
\end{aligned}
$$

In Sec. II we discussed how an approximate parallelogram may be chosen by observing the pattern of the stationary phase points in $\left(\bar{q}_{l}^{0}, \bar{q}_{j}^{0}\right)$ space. In Sec. III. A we associate $A, B, C$, and $D$ with $T_{1}, T_{5}, T_{10}$, and $T_{6}$, respectively, and hence obtain Eq. (3.9) from Eq. (C5)

The nume rical values of $K$ and $k$ obtained in Sec. III. A for the rather similarly shaped unit cell in Fig. 6 would be equal to those obtained by the rigorous methods desecribed by Eqs. (C2) and (C4). However, if the contours of the calculated phase become distorted to the point where one can no longer find (in Step 3B of the flow sheet) straight lines which more or less parallel the $u$ and $v$-curves and which give integer values of $k$ and $K$, it would be necessary to find the points $T_{1}, T_{5}, T_{8}$, and $T_{10}$ and to use Eqs. (C2) and (C4) to obtain the values of $K$ and $k$. In the systems studied thus far it was necessary only to use the approximate methods given in Step 3B. This circumstance has, of course, removed the need for determining the phases at the termination points, or for constructing the contour plots for each transition studied.

*Supported by a grant from the National Science Foundation. ${ }^{1}$ H. Kreek, R. L. Ellis, and R. A. Marcus, J. Chem. Phys 61, $\operatorname{xxxx}(1974)$.

${ }^{2}$ R. A. Marcus, J. Chem. Phys. 57, 4903 (1972).

${ }^{3}$ The exact results for $J=6$ are taken from B. R. Johnson, D. Secrest, and W. A. Lester, Chem. Phys. Lett. 1, 396 (1967), and those for the other $J$ 's from W. Eastes, J. Chem. Phys. 59, 3534 (1973).

${ }^{4}$ W. H. Miller, J. Chem. Phys. 54, 5386 (1971); J. D. Doll and W. H. Miller, J. Chem. Phys. 55, 3150 (1971).

${ }^{5}$ D. E. Fitz and R. A. Marcus, J. Chem. Phys. 59, 4380 (1973).

${ }^{6}$ R. A. Marcus, J. Chem. Phys. 56, 3548 (1972),

${ }^{7}$ W. H. Miller, J. Chem. Phys. 53, 3578 (1970).

${ }^{8} \mathrm{H}$. A. Scanton, Waves and Stability in Viscous and Enviscid Compressible Liquids Contained in Rigid and Elastic Maxima by the Method of Eigenvalues, Ph. D. dissertation, Carnegie-Mellon University, Pittsburg, 1970 (available from University Microfilms, Ann Arbor, Michigan, 48106, Order No. 70-18, 029E).

${ }^{9}$ Semiclassically, $\hat{j}$ equals $\left(j+\frac{1}{2}\right) \hbar$, etc. , using conventional units.

${ }^{10}$ H. Kreek and R. A. Marcus, J. Chem. Phys. 61, 3308 (1974) Addendum: Eq. (3.11) there was used for $i=j$. For $i=l$, the present (A6) and (A8) were used (without the $\frac{1}{2} \pi$ 's) which do not affect $\left|S_{m n}\right|^{2}$.

${ }^{11}$ (a) The terms $l^{f}, j^{f}, p_{R}^{f}, q_{l}(t)$, and $q_{f}(t)$ in Eq. (3. 3) are periodic functions of $\vec{q}_{l}^{0}$ and $\bar{q}_{j}^{0}$ with period equal to that of the unit cell, e.g. , $\pi$ for the homonuclear case, $2 \pi$ for the heteronuclear one. We denote this period by $\tau$. If $\bar{q}_{l}^{0}$ is increased by $\tau$, the first, second, third, fourth, and fifth terms are increased by $\left(l^{f}-l_{1}\right) \tau,-\tau\left(l^{f}-l_{1}\right), 0,0,0$. A similar remark applies to the effect of an increase in $\bar{q}_{j}^{0}$ by $\tau$. Thus, $\Delta_{\rho}$ is periodic in both $\bar{q}_{t}^{0}$ and $\bar{q}_{j}^{0}$ with a period $\tau$. (b) Since $l_{1}, l_{2}, j_{1}$, and $j_{2}$ are integers, exp $i \Delta_{L}$ is a periodic function of $\bar{q}_{i}^{f}$ and $\bar{q}_{j}^{f}$. Since $\bar{q}_{l}^{f}$ and $\bar{q}_{j}^{f}$ are peridoic functions of $\bar{q}_{i}^{0}$ and $\bar{q}_{j}^{0}$, exp $i \Delta_{L}$ is also.

${ }^{12}$ The form of the periodic terms in Eq. (3.7) may be found using Eqs. (A6) - (A9) and Hamiltonians equations for the system in Appendix A.

${ }^{13} \mathrm{H}$. Kreek, R. L. Ellis, and R. A. Marcus (to be published).

${ }^{14}$ R. A. Marcus, Chem. Phys. Lett. 7, 525 (1970); J. Chem. Phys, 54, 3965 (1971).

${ }^{15} \mathrm{H}$. Hancock, Theory of Maxima and Minima (Dover, New York, 1960), p. 23ff.

${ }^{16} \mathrm{~J}$. Stine and R. A. Marcus, J. Chem. Phys. 59, 5145 (1973).

${ }^{17}$ The Airy value in Ref. 4 for $\left(J, l_{2}, j_{2} ; l_{1} j_{1}\right)$ of $\left.6,10,4 ; 8,2\right)$ was 0.369 , which is very close to the Airy value in Table IV of 0.364 based on a wrong configuration. The Airy value based on the correct configuration is seen from Table I to be 0.482 . The Airy value for a $\left(J, l_{2}, j_{2} ; l_{1}, j_{1}\right)$ of $(6,2,4 ; 4$, 2 ) in Ref. 4 was 0.211 , compared with 0.245 in Table IV and 0.285 in Table $I$. Other wrong configurations can also occur.

${ }^{18} \mathrm{~J}$. N. L. Connor and R. A. Marcur, J. Chem. Phys. 55, 5636 (1971).

${ }^{19}$ J. N. L. Connor, Mol. Phys. 25, 181 (1973); J. N. L. Connor, Chem. Soc. Faraday Discuss. 55, 51 (1973).

${ }^{20}$ L. A. Pars, A Treatise on Analytical Dynamics (Wiley, New York, 1965), Chap. 29. See Fig. 29.12 for a description of the angles $q_{l}$ and $q_{j}$.

${ }^{21}$ These quantities, in (A6)-(A9) obtained using an analytical integration from $R_{1}$ to $R_{1}=\infty$ and from $R^{f}$ to $R^{f}=\infty$ to those used in Refs. 4 and 6.

${ }^{22}$ B. Nagel, Ark. Fys. 27, 181 (1964)

${ }^{23} \mathrm{We}$ make use of the results for the general periodic case, 
given in Sec. IV of Ref. 1.

${ }^{24}$ Based on the definitions of $u_{S^{\prime}}, u_{S}, v_{Q^{\prime}}$ and in Sec. II of Ref. 1 it may be shown that when $K$ is negative, $\zeta$ is negative. Thus, $K$ and $\zeta$ may be written as $-|K|$ and $-|\zeta|$ in the equation defining the Bessel function and its derivative [Eqs. (2.15) of Ref. 1]. A change of variable $\omega$ to $-\omega$ in this definition shows that $J_{K}(\zeta)$ and $J_{K}^{\prime}(\zeta)$ equal to $J_{|K|}(|\zeta|)$ and
$-J_{! K !}(|\zeta|)$, respectively, when $\zeta$ is real.

${ }^{25}$ We use Eqs. (2.27) and (2.81) of Ref. 1, together with the results in Sec. IV there. Later, for the case of complex points, the results in Sec. III there are used.

${ }^{26}$ Incidentally, $\Delta$ increases from $T_{1}$ of $T_{6}$ and $\Delta_{L}^{\prime}$ also increases However, in the region between $\boldsymbol{P}_{2}$ and $P_{3}$ the periodic term dominates and $\Delta$ decreases from $P_{2}$ to $P_{3}$. 